\title{
GEOMETRICALLY CONSISTENT MESH MODIFICATION
}

\author{
A. BONITO*, R.H. NOCHETTO ${ }^{\dagger}$, AND M.S. PAULETTI ${ }^{\ddagger}$
}

\begin{abstract}
A new paradigm of adaptivity is to execute refinement, coarsening, and smoothing of meshes on manifolds with incomplete information about their geometry and yet preserve position and curvature accuracy. We refer to this collectively as geometrically consistent mesh modification. We discuss the concept of discrete geometric consistency, show the failure of naive approaches, and propose and analyze a simple algorithm that is geometrically consistent and accuracy preserving.
\end{abstract}

Key words. Mesh modification, manifold, parametric finite elements, accuracy preserving, geometric consistency, refinement, coarsening, smoothing, mean curvature, Willmore.

1. Introduction. Adaptivity is a fundamental instrument in computational science and engineering. Its basic principle is to equidistribute the computational effort by increasing resolution near fine scale features (refinement), removing degrees of freedom where the local scale is relatively large (coarsening), and moving nodes to maintain mesh quality (mesh smoothing). These processes rely on the implicit assumption that one has access to the exact domain geometry at the discrete level; for example, when adding a new node to a boundary element its projection to the exact boundary is available for use. This is not the case for free boundary problems (FBP) for which the geometry is one of the main unknowns. This work is precisely concerned with the study of adaptive procedures for parametric finite element methods for FBP. In this context, there is a natural geometric constraint satisfied between position and vector curvature. This constraint may be difficult or impossible to satisfy discretely, thereby leading to geometric inconsistencies and ensuing numerical artifacts. Depending on the nature of the geometric problem at hand, these numerical artifacts may adversely affect, deteriorate or even halt computations.

A new paradigm in mesh adaptation is the execution of geometrically consistent refinement, coarsening, and mesh smoothing on manifolds with incomplete information about their geometry. Our goal is to

preserve the geometric relation between position and vector curvature (geo-

metric consistency) as well as their accuracy after mesh modification.

This paper discusses this paradigm and presents a novel geometrically consistent accuracy preserving algorithm for mesh modification along with its analysis. Let $\mathbf{x}$ be the position and $\mathbf{h}$ the vector curvature of a hypersurface $\gamma$ of dimension $d \geq 1$ in $\mathbb{R}^{d+1}$, not directly accessible. Let $(\Gamma, \mathbf{X}, \mathbf{H})$ be finite element approximations of degree $k \geq 1$ of $(\gamma, \mathbf{x}, \mathbf{h})$, where $\Gamma$ may not interpolate $\gamma$ and $\mathbf{X}$ may not be the position over $\Gamma$. Let $\epsilon, \delta>0$ be control parameters of mesh modification (Sections 4.2 and 4.3). Intuitively, $\epsilon$ measures the motion of nodes (in $W^{1, \infty}$ ) and $\delta$ quantifies interpolation errors due to changes in cardinality or location of dofs. If $(\Gamma, \mathbf{X}, \mathbf{H})$ is geometrically consistent (GC) (Definition 2.1), then the output $\left(\Gamma^{*}, \mathbf{X}^{*}, \mathbf{H}^{*}\right)$ of our Algorithm of

* Texas A\&M University, Department of Mathematics, TAMU 3368, College Station, TX 77843, USA (bonito@math.tamu.edu). Partially supported by NSF grants DMS-0807811 and DMS-0914977.

$\dagger$ University of Maryland, Department of Mathematics and Institute for Physical Science and Technology, College Park, MD 20742, USA (rhn@math.umd.edu). Partially supported by NSF grant DMS-0807811.

$\ddagger$ Texas A\&M University, Department of Mathematics, TAMU 3368, College Station, TX 77843, USA (pauletti@math.tamu.edu). Partially supported by NSF grant DMS-0807811 and by Award No. KUS-C1-016-04, made by King Abdullah University of Science and Technology (KAUST). 
Section 3.2 is GC and satisfies for any given $\epsilon, \delta$ (Theorem 4.4 in Section 4)

$$
\left|\left\|\nabla_{\gamma}(\mathbf{x}-\mathbf{X})\right\|_{L^{2}(\gamma)}-\left\|\left.\nabla_{\gamma}\left(\mathbf{x}-\mathbf{X}^{*}\right)\right|_{L^{2}(\gamma)} \mid \leq C(\epsilon+\delta)\right\| \mathbf{H} \|_{L^{2}(\gamma)}\right.
$$

and

$$
\left.|||\mathbf{h}-\mathbf{H}|\right|_{L^{2}(\gamma)}-\left\|\mathbf{h}-\left.\mathbf{H}^{*}\right|_{L^{2}(\gamma)} \mid \leq C(\epsilon+\delta)\right\| \mathbf{H} \|_{H^{1}(\gamma)},
$$

with $C>0$ a geometric constant. It is important to notice that (1.1)-(1.2) are not asymptotic nor they reflect any error improvement. They express the preservation of the geometric accuracy after mesh modification. The new surface mesh can then be exploited by the underlying geometric flow without suffering from undesirable numerical artifacts. This turns out to be crucial in dealing with curvature driven flows, most notably for those of order higher than two, for which curvature is needed explicitly; we discuss this issue in Section 5.

The paper is organized as follows. Section 2 starts by describing the notion of geometric consistency for smooth manifolds along with the inherent difficulties to enforce it. We resort to refinement of both curves and surfaces as a motivation, and discuss the ensuing accuracy deterioration. In Section 3 we briefly recall the notion of finite elements on manifolds, and present our algorithm. In Section 4 we introduce some basic facts from differential geometry and use them to derive quantitative estimates for mesh modifications; in particular we prove (1.1) and (1.2). We also interpret these estimates for the three basic mesh procedures: refinement, coarsening, and mesh smoothing. We conclude in Section 5 with applications of Algorithm 1. We examine the mean curvature and Willmore flows in Sections 5.1 and 5.2. We also introduce a practical procedure for geometric consistent initialization in Section 5.3, discuss related issues of polynomial degree mismatch in Section 5.4 and sensitivity to small time steps in Section 5.5. All simulations were implemented within ALBERTA [15].

2. Geometric Consistency: Motivation. Let $\gamma$ be an underlying smooth manifold of dimension $d \geq 1$ without boundary embedded in $\mathbb{R}^{d+1}$, which we know only approximately. Let $\mathbf{x}$ denote the position of $\gamma$ (i.e. $\mathbf{x}: \gamma \rightarrow \mathbb{R}^{d+1}$ is the identity on $\gamma$ ) and $\mathbf{h}:=h \boldsymbol{\nu}$ the vector curvature (i.e. $h$ is the sum of the principal curvatures or the total curvature and $\boldsymbol{\nu}$ is the outer unit normal of $\gamma$ ). Hereafter vectors and matrices are denoted in boldface. Considering manifolds without boundary is not necessary but preferred for clarity purposes.

Functions defined on a smooth surface $\gamma$ can be extended to a tubular neighborhood [8]. Let $f$ be $C^{1}$ scalar-valued function defined on $\gamma$ and $F$ its extension to $\mathbb{R}^{d+1}$. The tangential gradient of $f$ is defined as the orthogonal projection of $\nabla F$ to the tangent hyperplane of $\gamma$

$$
\nabla_{\gamma} f:=\left.(\mathbf{I}-\boldsymbol{\nu} \otimes \boldsymbol{\nu}) \nabla F\right|_{\gamma} .
$$

This definition is independent of the extension (see for example $[13,22]$ ). The tangential gradient of a $C^{1}$ vector-valued function $\mathbf{v}$ is the unique tensor $\nabla_{\gamma} \mathbf{v}$ such that $\left(\nabla_{\gamma} \mathbf{v}\right) \boldsymbol{a}=\nabla_{\gamma}(\mathbf{v} \cdot \boldsymbol{a})$ for all $\boldsymbol{a}$ in $\mathbb{R}^{d+1}$, i.e. the $i$-th column of $\nabla_{\gamma} \mathbf{v}$ is $\nabla_{\gamma} v_{i}$, where $v_{i}$ is the $i$-th component of $\mathbf{v}$. The tangential divergence $\operatorname{div}_{\gamma} \mathbf{v}:=I: \nabla_{\gamma} \mathbf{v}$ is the trace of $\nabla_{\gamma} \mathbf{v}$. Finally for $C^{2}(\gamma)$ scalar or vector-valued functions $f$, the surface Laplacian or Laplace-Beltrami operator is defined by $\Delta_{\gamma} f:=\operatorname{div}_{\gamma} \nabla_{\gamma} f$.

If we employ the sign convention that $h>0$ for a sphere, then $\mathbf{h}$ and $\mathbf{x}$ are related by the fundamental geometric identity $[13,11,7]$

$$
\mathbf{h}=-\Delta_{\gamma} \mathbf{x}
$$


To derive the weak form of (2.1) we multiply by $\varphi$ and integrate by parts to get

$$
\int_{\gamma} \mathbf{h} \cdot \boldsymbol{\varphi}=\int_{\gamma} \nabla_{\gamma} \mathbf{x}: \nabla_{\gamma} \boldsymbol{\varphi}, \quad \forall \varphi \in H^{1}(\gamma)
$$

where $\mathbf{x} \in H^{1}(\gamma), \mathbf{h} \in\left[H^{1}(\gamma)\right]^{\#}$, the dual of $H^{1}(\gamma)$ with zero meanvalue; we will assume further regularity later. Note that $A: B:=\operatorname{Tr}\left(A^{\top} B\right)$ and no boundary term appears because $\gamma$ has no boundary.

Discrete objects in space will be hereafter denoted with capitals. Let $\Gamma$ be a piecewise polynomial approximation to $\gamma$ of degree $k \geq 1$ (Definition 3.3). Finally, let $\mathbb{V}(\Gamma) \subset H^{1}(\Gamma)$ be a finite element space consisting of vector-valued $C^{0}$, not $C^{1}$, piecewise polynomials of degree $k \geq 1$ (see Section 3.1 for a precise definition). Relation (2.2) suggests the following definition of geometric consistency.

Definition 2.1 (Geometrically consistent triple). A triple $(\Gamma, \boldsymbol{X}, \boldsymbol{H})$ is said to be geometrically consistent $(G C)$ if $\boldsymbol{X}, \boldsymbol{H} \in \mathbb{V}(\Gamma)$ and the following relation holds

$$
\int_{\Gamma} \boldsymbol{H} \cdot \boldsymbol{\Phi}=\int_{\Gamma} \nabla_{\Gamma} \boldsymbol{X}: \nabla_{\Gamma} \boldsymbol{\Phi}, \quad \forall \boldsymbol{\Phi} \in \mathbb{V}(\Gamma)
$$

We point out that this notion is of geometric nature, and so complementary to but independent from accuracy. Both notions are crucial in dealing with geometric problems: if the triple $(\Gamma, \mathbf{X}, \mathbf{H})$ is $\mathrm{GC}$ and close to $(\gamma, \mathbf{x}, \mathbf{h})$, so should be its successor after mesh modification $\left(\Gamma^{*}, \mathbf{X}^{*}, \mathbf{H}^{*}\right)$. We now explore whether or not this is the case when the mesh modification is the refinement of the unit sphere, whose exact triple $(\gamma, \mathbf{x}, \mathbf{h})$ is fully accessible; coarsening and mesh smoothing are examined later in Sections 4.5.2 and 4.5.3. We let $\Gamma$ be a piecewise linear or quadratic interpolant of $\gamma$, over a quasi-uniform mesh, $\mathbf{X}$ be the identity over $\Gamma$ and $\mathbf{H}$ be the result of an explicit computation via (2.3). The refinement process consists of bisecting simplices and projecting the new nodes to the sphere. For $d=2$, the $L^{2}$-accuracy for $\mathbf{h}=2 \boldsymbol{\nu}$ for

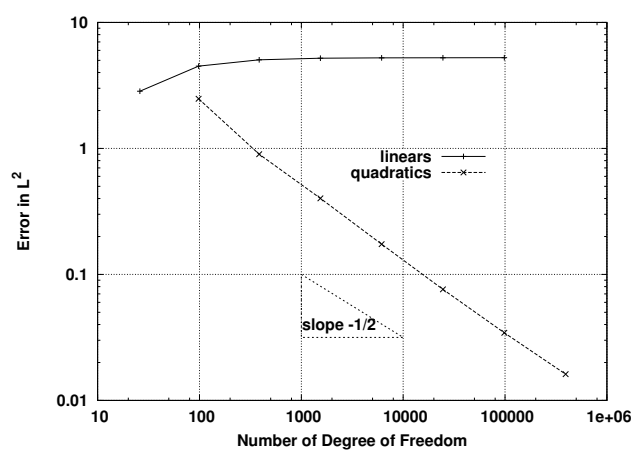

FIG. 2.1. Rate of convergence in $L^{2}(\Gamma)$ for the vector curvature $\boldsymbol{H}$ using (2.3) and identity $\boldsymbol{X}$ on a sequence of uniform refinements $\Gamma$ of the unit sphere $\gamma$. Piecewise linears do not converge whereas piecewise quadratics do, but with suboptimal order $\mathcal{N}^{-1 / 2}$.

the unit sphere is shown in Figure 2.1 for polynomial degrees $k=1,2$ and a sequence of uniform refinements. The order of convergence refers to the decay with respect to the number of degrees of freedom $\mathcal{N}$ : the optimal decay of the $L^{2}$-error for polynomial degree $k$ in dimension $d$ is $\mathcal{N}^{-(k+1) / d}$. Note that the refinement procedure adopted in this example is so that the $L^{2}$-error between the representations $\mathbf{x}$ of $\gamma$ and $\mathbf{X}$ of 
$\Gamma$ exhibits an optimal decay. We observe no order of convergence for the curvature $\mathbf{H}$ in $L^{2}(\Gamma)$ using linears $k=1$ and a suboptimal rate $\mathcal{N}^{-1 / 2}$ using quadratics $k=2$. This behavior is not surprising since it already happens for flat domains of dimension higher than 1. In this case, the Galerkin solution and the Lagrange interpolant differ at the nodes by an amount of order $\mathcal{N}^{-(k+1) / d}$. Computing the discrete Laplacian of the Lagrange interpolant entails two discrete derivatives and a corresponding order $\mathcal{N}^{-(k-1) / d}$, which is consistent with Figure 2.1.

Now let us consider the simpler 1D case $(d=1)$ but assuming that we have no access to the full geometry. Our best knowledge of the exact curve $\gamma$ is the current approximation $\Gamma$. Under these circumstances the simplest refinement that comes to mind is bisection of simplices without changing the curve $\Gamma$ or the identity $\mathbf{X}$. We refer to this as standard refinement procedure (STD). Figure 2.2 displays the behavior under refinement of a piecewise linear (top row) and piecewise quadratic (bottom row) uniform approximation of the unit circle $\gamma$ with four initial vertices. The curvature $\mathbf{H}$, satisfying (2.3) with $\mathbf{X}$ being the identity over $\Gamma$, is depicted in Figure 2.2(first column); the triples $(\Gamma, \mathbf{X}, \mathbf{H})$ are GC. Refining uniformly by bisection and keeping $\mathbf{X}$ as the identity over $\Gamma^{*}=\Gamma$ yields Figure 2.2(second column), which reveals lack of accuracy: in fact for linear elements the vector curvature $\mathbf{H}^{*}$ oscillates displaying a magnitude larger than 1 at the vertices and reverting direction at the midpoints - a devastating effect. This effect is less pronounced but noticeable for quadratic elements. This means that $\mathbf{H}^{*}$ is not as accurate as $\mathbf{H}$ to approximate $\mathbf{h}=\boldsymbol{\nu}$ in $L^{2}$, even though it is fine in $H^{-1}$. Figure 2.2(third and forth columns) show our geometrically consistent accuracy preserving (GCAP) method, which produces a GC triple $\left(\Gamma^{*}, \mathbf{X}^{*}, \mathbf{H}^{*}\right)$ that inherits the accuracy of $(\Gamma, \mathbf{X}, \mathbf{H})$. We present the method in Section 3.2 and analyze it in Section 4.
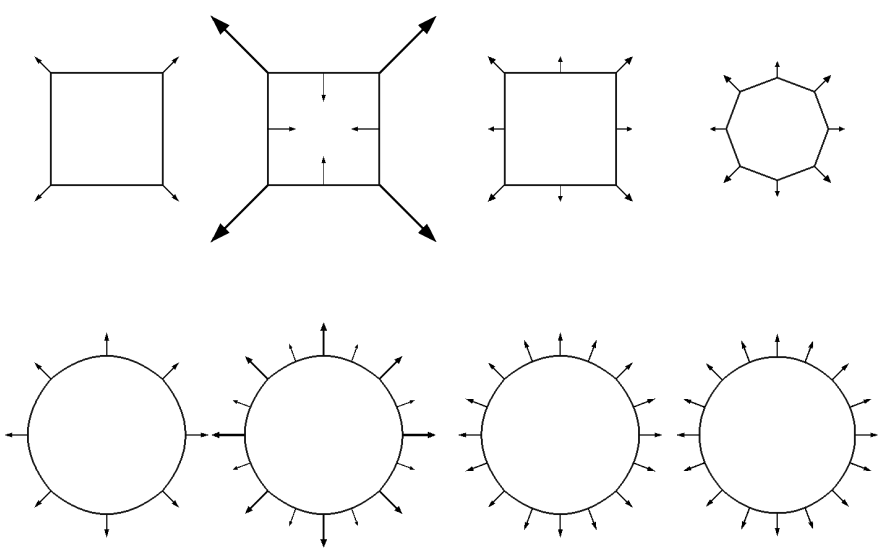

FIG. 2.2. Refinement procedures on a uniform partition of the unit circle $\gamma$ using linear (first row) and quadratic (second row) finite elements. The arrows on the piecewise polynomial curve $\Gamma$ represent the approximation $\boldsymbol{H}$ of the vector curvature $\boldsymbol{h}$, all scaled down by the same multiplicative factor 0.3. In each column we depict the original finite element curvature $\boldsymbol{H}$ (first), that after one global bisection of $\Gamma$ with $\boldsymbol{X}^{*}$ being the interpolant of $\boldsymbol{X}$ or simply the identity over $\Gamma^{*}$ (second), and that with our method (third). In contrast to our refinement, the former does not preserve the accuracy of the geometric approximations. The last picture (fourth) depicts the curvature $\boldsymbol{H}^{*}$ over the surface parametrized by $\boldsymbol{X}^{*}$ both obtained with our GCAP algorithm.

This behavior is not due to lack of resolution. Figure 2.3 illustrates the effect of 


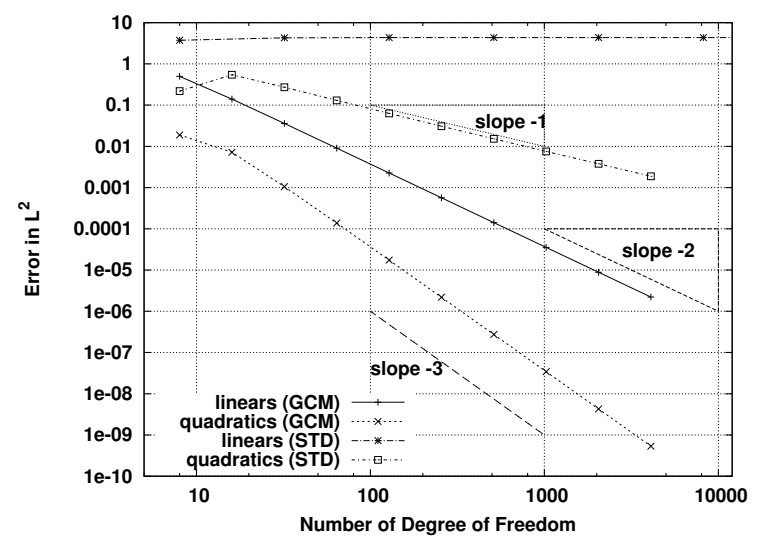

FIG. 2.3. Effect of one global bisection on a sequence of piecewise linear and quadratic approximation of the unit circle with decreasing meshsize. The $L^{2}$ error on the curvature is plotted vs the number of degree of freedom $\mathcal{N}$ after the standard refinement procedure (STD) and our geometrically consistent accuracy preserving (GCAP). The decay of the $L^{2}$-error on the curvature using STD for linears $k=1$ is $\mathcal{N}^{0}$ (no decrease) whereas that for quadratics $k=2$ is suboptimal $\mathcal{N}^{-1}$. In contrast, our GCAP method restores optimal error decay $\mathcal{N}^{-(k+1)}$.

one uniform bisection on a sequence of piecewise polynomial approximations of $\gamma$ of decreasing meshsize. We see that the $L^{2}$ error using piecewise linears for curvature does not decrease, whereas it exhibits an order one $\left(\mathcal{N}^{-1}\right)$ for piecewise quadratics. We also observe that our algorithm restores second order $\left(\mathcal{N}^{-2}\right)$ for linears and third order $\left(\mathcal{N}^{-3}\right)$ for quadratics, so that both benefit from the new approach.

To elucidate the strange behavior of linear finite elements we consider a piecewise linear curve $\Gamma$ made of straight segments $\Gamma_{i}$ connecting nodes $\mathbf{z}_{i}$ and $\mathbf{z}_{i+1}$. Let $\mathbf{X}$ be the identity on $\Gamma$ and $\mathbf{x}$ be a smooth curve passing through the nodes $\mathbf{z}_{i}$ of $\Gamma$. Since the tangential gradient and the derivative with respect to arclength $s$ are related as $\nabla_{\Gamma} \boldsymbol{\varphi}=\partial_{s} \boldsymbol{\varphi} \otimes \boldsymbol{\tau}$, where $\boldsymbol{\tau}$ is the unit tangent vector, we find that

$\int_{\Gamma} \partial_{s}(\mathbf{X}-\mathbf{x}) \cdot \partial_{s} \boldsymbol{\Phi}=-\sum_{i} \int_{\Gamma_{i}}(\mathbf{X}-\mathbf{x}) \cdot \partial_{s}^{2} \boldsymbol{\Phi}+\left.(\mathbf{X}-\mathbf{x}) \cdot \partial_{s} \boldsymbol{\Phi}\right|_{\mathbf{z}_{i}} ^{\mathbf{z}_{i+1}}=0, \quad \forall \boldsymbol{\Phi} \in \mathbb{V}(\Gamma)$,

because $\boldsymbol{\Phi}$ is linear on each $\Gamma_{i}$. Consequently, in view of (2.3), we deduce

$$
\int_{\Gamma} \mathbf{H} \cdot \mathbf{\Phi}=\int_{\Gamma} \partial_{s} \mathbf{X} \cdot \partial_{s} \mathbf{\Phi}=-\int_{\Gamma} \partial_{s}^{2} \mathbf{x} \cdot \mathbf{\Phi}, \quad \forall \mathbf{\Phi} \in \mathbb{V}(\Gamma),
$$

because $\mathbf{x}$ is smooth with respect to $s$. This shows that $\mathbf{H}$ is the $L^{2}$-projection of $\partial_{s}^{2} \mathbf{x}$ for any $\mathbf{x}$ that coincides with $\mathbf{X}$ at the nodes; thus $\mathbf{H}$ is close in $L^{2}$ to $\mathbf{h}$. Equivalently, $\mathbf{X}$ is the Ritz projection (and Lagrange interpolant) of $\mathbf{x}$, a result which is well known for piecewise linear finite elements in flat 1D domains.

This explanation has some important consequences. First, curvature $\mathbf{H}$ computed via (2.4), with $\mathbf{X}$ the Lagrange interpolant of $\mathbf{x}$, decays with optimal rate for curves. Second, we refer to Figure 2.4 to explain the effect of refinement reported in Figure 2.2 (top row). We may think of $\Gamma^{*}$ as a polygonal interpolating a new smooth curve $\gamma^{*}$ described by $\mathbf{x}^{*}$ (right), which is quite distinct from the unit circle $\gamma$ (left). The vector curvature $\mathbf{H}^{*}$, being the $L^{2}$-projection onto $\mathbb{V}(\Gamma)$ of $\partial_{s}^{2} \mathbf{x}^{*}$, oscillates and explains the behavior of the finite element solution $\mathbf{H}^{*}$. Therefore, the issue at stake is that placing 

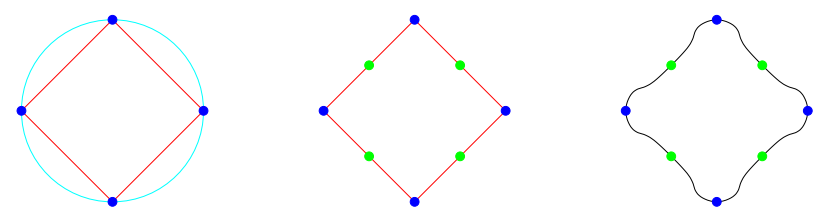

FIG. 2.4. Placing the new nodes created by bisection on the curve $\Gamma$ is equivalent to replacing the circle $\gamma$ being approximated (left) by a smooth curve (spline) $\gamma^{*}$ passing through all the nodes (right). This explains why the computed curvature $\boldsymbol{H}$ grows at the old nodes and changes direction at the new nodes. Compare with Figure 2.2(b, top row).

the new nodes over $\Gamma$, namely overrelying on the approximate curve, has undesirable geometric effects. The same happens for $d>1$. The question we will address later is how to exploit the incomplete information contained in the triple $(\Gamma, \mathbf{X}, \mathbf{H})$ to preserve its geometric consistency and accuracy while creating $\left(\Gamma^{*}, \mathbf{X}^{*}, \mathbf{H}^{*}\right)$.

The above explanation also sheds light on the rigid character of the geometric equation (2.3) if we were to enforce $\mathbf{X}$ to be the identity over $\Gamma$, which we do not! This is however a natural choice for initialization and the cause of severe problems for higher order flows for which both $\mathbf{H}$ and $\mathbf{X}$ are used in the formulation. Such problems have already been reported in the literature [3, 6, 12]. Dziuk proposes in [12] a procedure that is hard to implement and does not lead to $\mathbf{X}$ being the identity over $\Gamma$. In Section 5.3 we propose a simple and practical geometrically consistent method for initialization.

Further adverse effects of naive mesh modifications are documented in Section 4.5 .

3. Geometrically Consistent Accuracy Preserving Algorithm. To formulate (2.3) precisely, we introduce the notion of finite elements on manifolds along with some useful differential geometry concepts.

3.1. FE Approximation of Surfaces . We now introduce the notion of piecewise polynomial approximations of a smooth surface.

Definition 3.1 (Polyhedral surface). A pair $(\bar{\Gamma}, \overline{\mathcal{T}})$ is a polyhedral surface if $\bar{\Gamma} \subset \mathbb{R}^{d+1}$ and $\overline{\mathcal{T}}$ is a finite family of closed, non degenerate, $d$-simplices in $\mathbb{R}^{d+1}$ such that:

- the intersection of two simplices in the family is either empty or a sub-simplex of dimension $(d-k)$ of both simplices with $k=1, \ldots, d$;

- $\bar{\Gamma}=\bigcup_{\bar{K} \in \overline{\mathcal{T}}} \bar{K}$.

DeFInITIOn 3.2 (Polyhedral approximation and lift). Given a polyhedral surface $(\bar{\Gamma}, \overline{\mathcal{T}})$, a triple $(\bar{\Gamma}, \overline{\mathcal{T}}, \overline{\mathcal{L}})$ is a polyhedral approximation to $\gamma$ if the map $\overline{\mathcal{L}}: \bar{\Gamma} \rightarrow \gamma$ is a continuous bijection and $\left.\overline{\mathcal{L}}\right|_{\bar{K}}: \bar{K} \rightarrow \gamma$ is smooth for all $\bar{K} \in \overline{\mathcal{T}}$. The function $\overline{\mathcal{L}}$ is called lift and its smoothness will be specified below.

Notice that Definition 3.2 does not assume that nodes of $\overline{\mathcal{T}}$ lie on $\gamma$. Moreover, given $\gamma$ and $(\bar{\Gamma}, \overline{\mathcal{T}})$, there are infinitely many different lifts. A possible choice hinges on the signed distance function to $\gamma[10,7,9,21]$. However, there are instances, both theoretical and computational, when the distance function is not easily available (Mekchay et al. [17]).

To define higher order piecewise polynomial approximations to $\gamma$ we rely on the simplicial triple $(\bar{\Gamma}, \overline{\mathcal{T}}, \overline{\mathcal{L}})$ and the reference element in flat domain

$$
\widehat{K}:=\text { convex hull }\left(\boldsymbol{e}_{1}, \ldots, \boldsymbol{e}_{d}\right) \subset \mathbb{R}^{d} .
$$


Hence, given a polyhedral surface $(\bar{\Gamma}, \overline{\mathcal{T}})$, for each $\bar{K} \in \overline{\mathcal{T}}$ we let $\mathbf{F}_{\bar{K}}: \widehat{K} \rightarrow \bar{K}$ be the unique affine map that maps the vertices of $\widehat{K}$ to the corresponding ones of $\bar{K}$.

Definition 3.3 (Piecewise polynomial approximation of $\gamma$ ). A set $\Gamma \subset \mathbb{R}^{d+1}$ is a piecewise polynomial approximation of degree $k$ to a surface $\gamma$ if there exist

- a polyhedral approximation $(\bar{\Gamma}, \overline{\mathcal{T}}, \overline{\mathcal{L}})$ to $\gamma$,

- injective polynomial functions $\mathbf{F} \frac{k}{K}: \widehat{K} \rightarrow \mathbb{R}^{d+1}$ of degree $k$ for each $\bar{K} \in \overline{\mathcal{T}}$, such that the function $\mathcal{L}: \Gamma \rightarrow \gamma$ defined by $\left.\mathcal{L}\right|_{K}=\overline{\mathcal{L}} \circ \mathbf{F}_{\bar{K}} \circ\left(\mathbf{F}_{\bar{K}}^{k}\right)^{-1}$ is a continuous bijection.

We define $\mathcal{T}$ to be the set of isoparametric simplices $K=\mathbf{F} \frac{k}{K}(\widehat{K})$ of degree $k$ and the finite element space $\mathbb{V}(\Gamma)$ of vector-valued functions on $\Gamma$ to be

$$
\mathbb{V}(\Gamma):=\left\{\boldsymbol{\Phi} \in C^{0}\left(\Gamma ; \mathbb{R}^{d+1}\right): \boldsymbol{\Phi} \circ \mathbf{F}_{K}^{k} \in \mathbb{P}^{k}(\widehat{K})\right\},
$$

where $\mathbb{P}^{k}(\widehat{K})$ is the set of polynomial of degree $\leq k$ in $d$ variables. Observe that we have the same polynomial degree $k$ for the mesh $\mathcal{T}$ and the function space. Polynomial mismatch is discussed in Section 5.4.

3.2. GCAP Algorithm. Let $(\Gamma, \mathbf{X}, \mathbf{H})$ be a geometrically consistent triple and $\mathcal{T}$ be a finite element mesh describing the piecewise polynomial manifold $\Gamma$. Let $\mathcal{M}$ denote the subset of elements of $\mathcal{T}$ marked for either refinement, coarsening or mesh smoothing. The procedure TripleModify $(\Gamma, \mathbf{X}, \mathbf{H}, \mathcal{M})$ described in Algorithm 1 carries out the mesh modification.

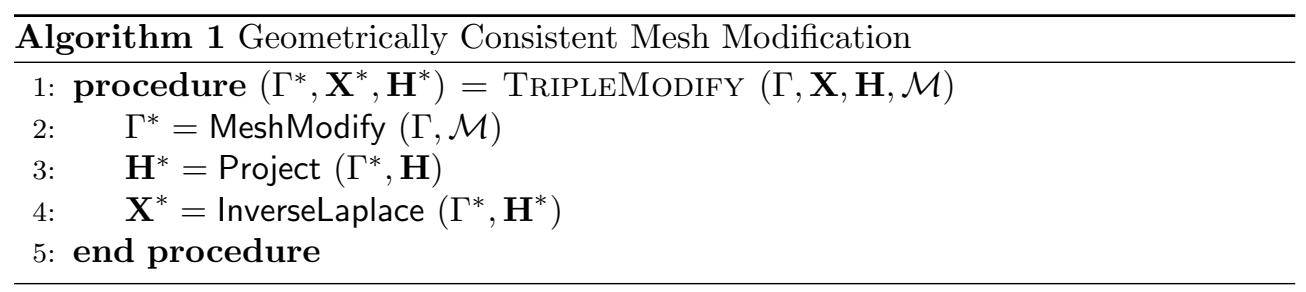

We now explain the three main modules separately and discuss a variant that conserves volume.

3.2.1. Module $\Gamma^{*}=$ MeshModify $(\Gamma, \mathcal{M})$. We construct the new manifold $\Gamma^{*}$ upon performing the mesh modification on the marked elements listed in $\mathcal{M}$. The addition, deletion, or relocation of nodes of an isoparametric mesh is handled by means of isoparametric interpolation techniques; see Section 4.5. Having the discrete manifolds $\Gamma$ and $\Gamma^{*}$, we relate them via the mesh modification function

$$
\boldsymbol{m}: \Gamma \rightarrow \Gamma^{*}
$$

we refer to Section 4.2 for details. Refinement of $\gamma$ implies that $\Gamma^{*}=\Gamma$ and $\boldsymbol{m}=i d_{\Gamma}$ : the surfaces coincide but the number of elements used to represent $\Gamma^{*}$ is larger than that for $\Gamma$. The opposite occurs for coarsening whereas no change in the number of elements happens for mesh smoothing, and in both cases $\Gamma^{*} \neq \Gamma$.

3.2.2. Module $\mathbf{H}^{*}=$ Project $\left(\Gamma^{*}, \mathbf{H}\right)$. This module computes the $L^{2}$ projection $\mathbf{H}^{*}$ of $\mathbf{H}$ onto the new finite element space $\mathbb{V}\left(\Gamma^{*}\right)$ over $\Gamma^{*}$ as follows:

$$
\mathbf{H}^{*} \in \mathbb{V}\left(\Gamma^{*}\right): \quad \int_{\Gamma^{*}} \mathbf{H}^{*} \cdot \boldsymbol{\Phi}^{*}=\int_{\Gamma} \mathbf{H} \cdot\left(\boldsymbol{\Phi}^{*} \circ \boldsymbol{m}\right), \quad \forall \boldsymbol{\Phi}^{*} \in \mathbb{V}\left(\Gamma^{*}\right) .
$$

We note that $\mathbf{H}^{*}=\mathbf{H}$ for refinement since $\Gamma \equiv \Gamma^{*}$ and thus $\mathbb{V}(\Gamma) \subset \mathbb{V}\left(\Gamma^{*}\right)$. 
3.2.3. Module $\mathbf{X}^{*}=$ InverseLaplace $\left(\Gamma^{*}, \mathbf{H}^{*}\right)$. This module computes the new position $\mathbf{X}^{*}$ as the solution to

$$
\mathbf{X}^{*} \in \mathbb{V}\left(\Gamma^{*}\right): \quad \int_{\Gamma^{*}} \nabla_{\Gamma^{*}} \mathbf{X}^{*}: \nabla_{\Gamma^{*}} \mathbf{\Phi}^{*}=\int_{\Gamma^{*}} \mathbf{H}^{*} \cdot \boldsymbol{\Phi}^{*}, \quad \forall \boldsymbol{\Phi}^{*} \in \mathbb{V}\left(\Gamma^{*}\right)
$$

subject to the mean value condition $\int_{\Gamma} \mathbf{X}=\int_{\Gamma^{*}} \mathbf{X}^{*}$. Note that the existence of $\mathbf{X}^{*}$ is ensured by the compatibility condition

$$
\int_{\Gamma^{*}} \mathbf{H}^{*}=0
$$

which in turn is due to the geometric consistency $(2.3)$ of $(\Gamma, \mathbf{X}, \mathbf{H})$ and the definition (3.3) of $\mathbf{H}^{*}$. Relation (3.4) defining $\mathbf{X}^{*}$ guarantees that the triple $\left(\Gamma^{*}, \mathbf{X}^{*}, \mathbf{H}^{*}\right)$ is GC in the sense of Definition 2.1.

3.2.4. GCAP with Volume Conservation. If $\Gamma^{*}=\Gamma$ then volume is trivially conserved (for e.g. refinement). We point out that we can easily enforce volume conservation whenever $\Gamma^{*} \neq \Gamma$ (for e.g. coarsening and mesh smoothing). If $\mathbf{Y}$ denotes the identity over $\Gamma$, and $\boldsymbol{\nu}$ is the unit outer normal, then the volume enclosed by $\Gamma$ can be written as $\frac{1}{d+1} \int_{\Gamma} \mathbf{Y} \cdot \boldsymbol{\nu}$ with the help of the divergence theorem. Likewise, the volume enclosed by $\Gamma^{*}$ is given by $\frac{1}{d+1} \int_{\Gamma^{*}} \mathbf{Y}^{*} \cdot \boldsymbol{\nu}^{*}$, whence the adequate volume scaling factor $\alpha>0$ is

$$
\alpha=\left(\frac{\int_{\Gamma} \mathbf{Y} \cdot \boldsymbol{\nu}}{\int_{\Gamma^{*}} \mathbf{Y}^{*} \cdot \boldsymbol{\nu}^{*}}\right)^{\frac{1}{d+1}}
$$

In fact, it is easy to check that the following triple $\left(\Gamma^{* *}, \mathbf{X}^{* *}, \mathbf{H}^{* *}\right)$

$$
\Gamma^{* *}=\alpha \Gamma^{*}, \quad \mathbf{X}^{* *}=\alpha \mathbf{X}^{*}, \quad \mathbf{H}^{* *}=\frac{1}{\alpha} \mathbf{H}^{*},
$$

is GC. Moreover, $\boldsymbol{\nu}^{* *}\left(\mathbf{Y}^{* *}\right)=\boldsymbol{\nu}^{*}\left(\mathbf{Y}^{*}\right)$ implies $\int_{\Gamma^{* *}} \mathbf{Y}^{* *} \cdot \boldsymbol{\nu}^{* *}=\int_{\Gamma} \mathbf{Y} \cdot \boldsymbol{\nu}$ whence the volume is conserved. For this volume preserving mesh modification, $\alpha \rightarrow 1$ as the meshsize goes to 0 , thus maintaining the inherent accuracy of $(\Gamma, \mathbf{X}, \mathbf{H})$.

4. Quantitative Mesh Modification. In this section we introduce a quantitative measure for mesh modification and prove a bound on the accuracy of the GCAP Algorithm in terms of the mesh modification parameters $\epsilon$ and $\delta$ characterized by (4.5) and (4.6) respectively.

4.1. Differential Geometry. We obtain formulas to relate differentials over different domains. The differentials generalize the classical properties from calculus on open sets in Euclidean space to surfaces. In particular, the chain rule, implicit function Theorem and inverse function Theorem are satisfied under the expected assumptions. Here we briefly mention some facts and concepts and refer the reader to any reasonable differential geometry book like [18] for details.

Given an oriented surface $S$ and a point $p \in S, \boldsymbol{\nu}(p)$ denotes the unit normal vector of $S$ at $p$ and $T_{p} S$ denotes the tangent plane of $S$ at $p$, which is the subspace of $\mathbb{R}^{d+1}$ orthogonal to $\boldsymbol{\nu}(p)$. 
4.1.1. Differentials and Surface Gradients. Given two regular surfaces $S_{1}$ and $S_{2}$ and a differentiable map $g: S_{1} \rightarrow S_{2}$, d $g$ denotes its differential. For $p \in S_{1}$, $\mathrm{d} g(p): T_{p} S_{1} \rightarrow T_{g(p)} S_{2}$ is a linear map between tangent planes. We define

$$
|\mathrm{d} g(p)|:=\sup _{\left\{\boldsymbol{t} \in T_{p} S_{1},|\boldsymbol{t}|=1\right\}}|\mathrm{d} g(p)[\boldsymbol{t}]|
$$

to be the operator norm, where the norms in $T_{p} S_{1}$ and $T_{g(p)} S_{2}$ are euclidean norms inherited as the restriction of the euclidian product in $\mathbb{R}^{d+1}$ to the tangent planes.

If $f: S_{1} \rightarrow \mathbb{R}$ is differentiable then $\mathrm{d} f(p)[\boldsymbol{t}]=\nabla_{S_{1}} f(p)^{\top} \boldsymbol{t}$, and if $\boldsymbol{f}: S_{1} \rightarrow \mathbb{R}^{m}$ is differentiable then $\mathrm{d} \boldsymbol{f}(p)=\nabla_{S_{1}} \boldsymbol{f}(p)^{\top}$, thereby making a link with the definition of $\nabla_{S_{1}} \boldsymbol{f}(p)$ by columns given in Section 2. Finally for $\boldsymbol{\phi}: S_{1} \rightarrow S_{2}$ we set

$$
\nabla_{S_{1}} \phi(p):=\mathrm{d} \phi(p)^{\top} .
$$

4.1.2. Change of Variables. If $\phi: S_{1} \rightarrow S_{2}$ is a diffeomorphism and $\boldsymbol{w}: S_{1} \rightarrow$ $\mathbb{R}^{m}$ is differentiable then $d\left(\boldsymbol{w} \circ \boldsymbol{\phi}^{-1}\right)(\boldsymbol{\phi}(x))=d \boldsymbol{w}(x) d \boldsymbol{\phi}^{-1}(\boldsymbol{\phi}(x))$, whence

$$
\nabla_{S_{2}}\left(\boldsymbol{w} \circ \phi^{-1}\right)(\phi(x))=\nabla_{S_{2}} \boldsymbol{\phi}^{-1}(\phi(x)) \nabla_{S_{1}} \boldsymbol{w}(x) .
$$

If $f: S_{1} \rightarrow \mathbb{R}$ is differentiable then

$$
\int_{S_{2}} f=\int_{S_{1}}(f \circ \phi)|\operatorname{det}(\mathrm{d} \phi)| .
$$

Observe that for $d=1,|\operatorname{det}(\mathrm{d} \phi)(p)|=\left|\mathrm{d} \boldsymbol{\phi}(p)\left[\boldsymbol{e}_{1}\right]\right|$ where $\boldsymbol{e}_{1}$ is a unit vector in $T_{p} S$, whereas for $d=2,|\operatorname{det}(\mathrm{d} \phi)(x)|=\left|\mathrm{d} \phi(x)\left[\boldsymbol{e}_{1}\right] \times \mathrm{d} \boldsymbol{\phi}(x)\left[\boldsymbol{e}_{2}\right]\right|$ where $\boldsymbol{e}_{1}$ and $\boldsymbol{e}_{2}$ are two orthonormal vectors in $T_{p} S$. Therefore, the bound

$$
|\operatorname{det}(\mathrm{d} \phi)| \leq|\mathrm{d} \phi|^{d}
$$

holds for $d=1,2$, and for $d>2$ a similar reasoning applies using the definition of cross product for higher dimensions (see [23, Ch.4]).

4.2. Measure of Mesh Modification. We recall that a mesh modification is a transformation $\boldsymbol{m}: \Gamma \rightarrow \Gamma^{*}$ from a piecewise polynomial approximation $\Gamma$ of $\gamma$ into another one $\Gamma^{*}$. It is the purpose of this section to quantify the effect of $\boldsymbol{m}$.

To be precise we first define the sets of extended elements $\mathcal{T}_{e}$ and $\mathcal{T}_{e}^{*}$ of $\Gamma$ and $\Gamma^{*}$ as follows. If $K \in \mathcal{T}$ is not affected by refinement then $K \in \mathcal{T}_{e}$, else if $K$ is refined and gives elements in $\mathcal{T}^{*}$ then the corresponding subdivisions (subsets) of $K$ are extended elements in $\mathcal{T}_{e}$. If $K^{*} \in \mathcal{T}^{*}$ is not the result of coarsening then $K^{*} \in \mathcal{T}_{e}^{*}$, else the corresponding subdivisions of $K^{*}$ are extended elements in $\mathcal{T}_{e}^{*}$. In this way there is a natural bijection between the elements of $\mathcal{T}_{e}$ and those of $\mathcal{T}_{e}^{*}$ that we use to define the notion of mesh modification. Examples are given in Section 4.5.

Definition 4.1 (Mesh modification). A homeomorphism $\boldsymbol{m}: \Gamma \rightarrow \Gamma^{*}$ is a mesh modification if $\boldsymbol{m}: K \rightarrow K^{*}$ is a diffeomorphism for each $K \in \mathcal{T}_{e}$ and its corresponding $K^{*} \in \mathcal{T}_{e}^{*}$.

We use the convention that functions with domain in $\Gamma$ appearing inside integrals or norms over $\Gamma^{*}$ are understood as composed with $\boldsymbol{m}^{-1}$, and viceversa for functions with domain $\Gamma^{*}$. The manifolds $\Gamma$ and $\Gamma^{*}$ are piecewise smooth and globally Lipschitz. For Lipschitz manifolds it is possible to define $H^{1}(\Gamma)$ and show that $C^{0,1}(\Gamma) \subset H^{1}(\Gamma)$, whence for $\boldsymbol{\Phi} \in \mathbb{V}(\Gamma)$ we have $\left.\nabla_{\Gamma} \boldsymbol{\Phi}\right|_{K}=\nabla_{K} \boldsymbol{\Phi}$ (see [25, Sec 4.2]). 
If $\operatorname{Id}_{\Gamma}$ and $\operatorname{Id}_{\Gamma^{*}}$ denote the identity functions over $\Gamma$ and $\Gamma^{*}$, then it is trivial to see that $\nabla_{\Gamma} \operatorname{Id}_{\Gamma}=\mathbf{I}$ and $\nabla_{\Gamma^{*}} \operatorname{Id}_{\Gamma^{*}}=\mathbf{I}^{*}$, where $\mathbf{I}(x): T_{x} \Gamma \rightarrow T_{x} \Gamma$ and $\mathbf{I}^{*}(x): T_{x^{*}} \Gamma^{*} \rightarrow$ $T_{x^{*}} \Gamma^{*}$ are the identities on the tangent planes of $\Gamma$ at $x$ and $\Gamma^{*}$ at $x^{*}$.

To simplify the notation let $\sigma=|\operatorname{det}(\mathrm{d} \boldsymbol{m})|, \mathbf{N}(x)=\sigma^{1 / 2}(x) \nabla_{\Gamma^{*}} \boldsymbol{m}^{-1}(\boldsymbol{m}(x))$ and $\mathbf{M}\left(x^{*}\right)=\sigma^{-1 / 2}\left(\boldsymbol{m}^{-1}\left(x^{*}\right)\right) \nabla_{\Gamma} \boldsymbol{m}\left(\boldsymbol{m}^{-1}\left(x^{*}\right)\right)$. Then $\mathbf{M}$ and $\mathbf{N}$ are inverses as

$$
\mathbf{M}(\boldsymbol{m}(x)) \mathbf{N}(x)=\mathbf{I}(x) \quad \text { and } \quad \mathbf{N}\left(\boldsymbol{m}^{-1}\left(x^{*}\right)\right) \mathbf{M}\left(x^{*}\right)=\mathbf{I}^{*}\left(x^{*}\right) .
$$

Invoking the change of variable formulas (4.1) and (4.2) we get

$$
\begin{array}{ll}
\int_{\Gamma^{*}} \nabla_{\Gamma^{*}} \boldsymbol{\Phi}: \nabla_{\Gamma^{*}} \boldsymbol{\Psi}=\int_{\Gamma} \mathbf{N}^{\top} \mathbf{N} \nabla_{\Gamma} \boldsymbol{\Phi}: \nabla_{\Gamma} \boldsymbol{\Psi}, & \int_{\Gamma^{*}} \phi=\int_{\Gamma} \phi \sigma, \\
\int_{\Gamma} \nabla_{\Gamma} \boldsymbol{\Phi}: \nabla_{\Gamma} \boldsymbol{\Psi}=\int_{\Gamma^{*}} \mathbf{M}^{\top} \mathbf{M} \nabla_{\Gamma^{*}} \boldsymbol{\Phi}: \nabla_{\Gamma^{*}} \boldsymbol{\Psi}, & \int_{\Gamma} \phi=\int_{\Gamma^{*}} \phi \sigma^{-1} .
\end{array}
$$

Upon controlling the changes on the tangent planes due to $\boldsymbol{m}$, (4.4) allows us to quantify the errors incurred. The orthogonal projections onto $\Gamma$ and $\Gamma^{*}$ are

$$
\mathbf{P}:=\mathbf{I}_{d+1}-\boldsymbol{\nu} \otimes \boldsymbol{\nu}, \quad \mathbf{P}^{*}:=\mathbf{I}_{d+1}-\boldsymbol{\nu}^{*} \otimes \boldsymbol{\nu}^{*} .
$$

Lemma 4.2 (Effect of mesh modification). Let $\Gamma$ be a piecewise polynomial approximation of degree $k$ to a surface $\gamma$. Let $\boldsymbol{m}: \Gamma \rightarrow \Gamma^{*}$ be a mesh modification in the sense of Definition 4.1 satisfying

$$
\left\|\mathbf{P} \nabla_{\Gamma} \boldsymbol{m} \mathbf{P}^{*}-\mathbf{P P}^{*}\right\|_{L^{\infty}(\Gamma)}+\left\|\mathbf{P}^{*} \nabla_{\Gamma^{*}} \boldsymbol{m}^{-1} \mathbf{P}-\mathbf{P}^{*} \mathbf{P}\right\|_{L^{\infty}\left(\Gamma^{*}\right)} \leq \epsilon,
$$

for some $0<\epsilon \leq \epsilon_{0}$. Then, there exist a constant $C>0$ only depending on $d$ and $\epsilon_{0}$, such that for all $\boldsymbol{\Phi} \in L^{2}(\Gamma)$ and $\boldsymbol{\Psi} \in L^{2}(\Gamma)$ there holds

$$
\left|\int_{\Gamma} \boldsymbol{\Phi} \cdot \boldsymbol{\Psi}-\int_{\Gamma^{*}} \boldsymbol{\Phi} \cdot \boldsymbol{\Psi}\right| \leq C \epsilon\|\boldsymbol{\Phi}\|_{L^{2}(\Gamma)}\|\boldsymbol{\Psi}\|_{L^{2}(\Gamma)}
$$

and for all $\mathbf{\Phi} \in H^{1}(\Gamma)$ and $\boldsymbol{\Psi} \in H^{1}(\Gamma)$ there holds

$$
\left|\int_{\Gamma} \nabla_{\Gamma} \boldsymbol{\Phi}: \nabla_{\Gamma} \boldsymbol{\Psi}-\int_{\Gamma^{*}} \nabla_{\Gamma^{*}} \boldsymbol{\Phi}: \nabla_{\Gamma^{*}} \boldsymbol{\Psi}\right| \leq C \epsilon\left\|\nabla_{\Gamma} \boldsymbol{\Phi}\right\|_{L^{2}(\Gamma)}\left\|\nabla_{\Gamma} \boldsymbol{\Psi}\right\|_{L^{2}(\Gamma)} .
$$

Proof. Let $C>0$ denote a generic constant only depending on $d$. First we show that (4.5) implies that $\left\|\nabla_{\Gamma} \boldsymbol{m}\right\|_{L^{\infty}(\Gamma)} \leq(1+\epsilon)$. To see this observe that $\|\mathbf{P}\|_{2}=1$, $\mathbf{P} \nabla_{\Gamma} \boldsymbol{m}=\nabla_{\Gamma} \boldsymbol{m}$, and $\mathbf{P}^{*} \boldsymbol{t}^{*}=\boldsymbol{t}^{*}$ for any unit vector $\boldsymbol{t}^{*} \in T_{\boldsymbol{m}(x)} \Gamma^{*}$, whence

$$
\nabla_{\Gamma} \boldsymbol{m}(x) \boldsymbol{t}^{*}=\mathbf{P} \boldsymbol{t}^{*}+\mathbf{P} \nabla_{\Gamma} \boldsymbol{m} \mathbf{P}^{*} \boldsymbol{t}^{*}-\mathbf{P} \mathbf{P}^{*} \boldsymbol{t}^{*}
$$

and $\left\|\nabla_{\Gamma} \boldsymbol{m}\right\|_{L^{\infty}(\Gamma)} \leq(1+\epsilon)$; similarly $\left\|\nabla_{\Gamma^{*}} \boldsymbol{m}^{-1}\right\|_{L^{\infty}\left(\Gamma^{*}\right)} \leq(1+\epsilon)$. This implies

$$
\sigma=|\operatorname{det}(\mathrm{d} \boldsymbol{m})| \leq\left\|\nabla_{\Gamma} \boldsymbol{m}\right\|_{L^{\infty}(\Gamma)}^{d} \leq(1+\epsilon)^{d} \leq 1+C \epsilon,
$$

as well as $\sigma^{-1} \leq 1+C \epsilon$. These two inequalities yield

$$
\|1-\sigma\|_{L^{\infty}(\Gamma)} \leq C \epsilon .
$$

In order to use (4.4), we let $\widetilde{\mathbf{N}}=\sigma^{-1 / 2} \mathbf{N}=\nabla_{\Gamma^{*}} \boldsymbol{m}^{-1}$ and $\widetilde{\mathbf{M}}=\sigma^{1 / 2} \mathbf{M}=\nabla_{\Gamma} \boldsymbol{m}$, and note that $\mathbf{I}-\mathbf{N}^{\top} \mathbf{N}=(1-\sigma) \mathbf{I}+\sigma\left(\mathbf{I}-\widetilde{\mathbf{N}}^{\top} \widetilde{\mathbf{N}}\right)$. We rewrite the last term as

$$
\mathbf{I}-\widetilde{\mathbf{N}}^{\top} \widetilde{\mathbf{N}}=\widetilde{\mathbf{M}} \widetilde{\mathbf{N}}-\widetilde{\mathbf{N}}^{\top} \widetilde{\mathbf{N}}=\left(\widetilde{\mathbf{M}}-\widetilde{\mathbf{N}}^{\top}\right) \widetilde{\mathbf{N}}
$$


Since $\mathrm{d} \boldsymbol{m}(p)[\boldsymbol{t}]=\boldsymbol{t}^{*} \in T_{m(p)} \Gamma^{*}$ for all $\boldsymbol{t} \in T_{p} \Gamma$, we deduce $\mathbf{P}^{*} \mathrm{~d} \boldsymbol{m}(p)=\mathrm{d} \boldsymbol{m}(p)$ or equivalently $\widetilde{\mathbf{M}} \mathbf{P}^{*}=\widetilde{\mathbf{M}}$; likewise $\mathbf{P} \widetilde{\mathbf{N}}^{\top}=\widetilde{\mathbf{N}}^{\top}$. This gives

$$
\left(\widetilde{\mathbf{M}}-\widetilde{\mathbf{N}}^{\top}\right)=\mathbf{P}\left(\widetilde{\mathbf{M}}-\widetilde{\mathbf{N}}^{\top}\right) \mathbf{P}^{*}=\left(\mathbf{P} \widetilde{\mathbf{M}} \mathbf{P}^{*}-\mathbf{P} \mathbf{P}^{*}\right)-\left(\mathbf{P} \widetilde{\mathbf{N}}^{\top} \mathbf{P}^{*}-\mathbf{P} \mathbf{P}^{*}\right)
$$

as well as $\left\|\widetilde{\mathbf{M}}-\widetilde{\mathbf{N}}^{\top}\right\|_{L^{\infty}(\Gamma)} \leq 2 \epsilon$, according to (4.5). We finally realize that

$$
\left\|\mathbf{I}-\mathbf{N}^{\top} \mathbf{N}\right\|_{L^{\infty}(\Gamma)} \leq C \epsilon+2 \epsilon(1+C \epsilon),
$$

and the assertion follows from (4.4).

We end this section by providing a norm equivalence property.

LEMma 4.3 (Norm equivalence). Let $\Gamma$ be a piecewise polynomial approximation of degree $k$ to a surface $\gamma$. Then there exists constants $0<c<C$, only depending on the associated lift $\mathcal{L}$, such that for all $\phi \in L^{2}(\Gamma)$

$$
c\|\phi\|_{L^{2}(\gamma)} \leq\|\phi\|_{L^{2}(\Gamma)} \leq C\|\phi\|_{L^{2}(\gamma)},
$$

and for all $\phi \in H^{1}(\Gamma)$

$$
c\|\phi\|_{H^{1}(\gamma)} \leq\|\phi\|_{H^{1}(\Gamma)} \leq C\|\phi\|_{H^{1}(\gamma)} .
$$

Proof. Proceed as in Lemma 4.2, replacing $\Gamma^{*}$ by $\gamma$ and $\boldsymbol{m}$ by $\mathcal{L}$. $\square$

4.3. Interpolation Operators. We define the Ritz or elliptic projection operator $\Pi^{*}: H^{1}\left(\Gamma^{*}\right) \rightarrow \mathbb{V}\left(\Gamma^{*}\right)$ as follows: for all $\boldsymbol{\Psi} \in H^{1}\left(\Gamma^{*}\right)$, let $\Pi^{*} \boldsymbol{\Psi} \in \mathbb{V}\left(\Gamma^{*}\right)$ satisfy

$$
\int_{\Gamma^{*}} \nabla_{\Gamma^{*}}\left(\Pi^{*} \boldsymbol{\Psi}-\boldsymbol{\Psi}\right): \nabla_{\Gamma^{*}} \boldsymbol{\Phi}^{*}=0 \quad \forall \boldsymbol{\Phi}^{*} \in \mathbb{V}\left(\Gamma^{*}\right), \quad \int_{\Gamma^{*}}\left(\Pi^{*} \boldsymbol{\Psi}-\mathbf{\Psi}\right)=0 ;
$$

such $\Pi^{*} \boldsymbol{\Psi}$ is unique. Similarly $\Pi: H^{1}(\Gamma) \rightarrow \mathbb{V}(\Gamma)$ satisfies for all $\boldsymbol{\Psi} \in H^{1}(\Gamma)$

$$
\int_{\Gamma} \nabla_{\Gamma}(\Pi \Psi-\boldsymbol{\Psi}): \nabla_{\Gamma} \boldsymbol{\Phi}=0 \quad \forall \boldsymbol{\Phi} \in \mathbb{V}(\Gamma), \quad \int_{\Gamma}(\Pi \Psi-\boldsymbol{\Psi})=0 .
$$

Let $(\Gamma, \mathbf{X}, \mathbf{H})$ and $\left(\Gamma^{*}, \mathbf{X}^{*}, \mathbf{H}^{*}\right)$ be the triples before and after the mesh modification. Our main result relies on the existence of a parameter $\delta>0$ such that

$$
\begin{aligned}
\left|\int_{\Gamma} \mathbf{H} \cdot\left(\mathbf{X}-\Pi^{*} \mathbf{X}\right)\right| & \leq \delta^{2}\|\mathbf{H}\|_{L^{2}(\Gamma)}\left\|\nabla_{\Gamma} \mathbf{X}\right\|_{L^{2}(\Gamma)}, \\
\left|\int_{\Gamma} \mathbf{H} \cdot\left(\mathbf{X}^{*}-\Pi \mathbf{X}^{*}\right)\right| & \leq \delta^{2}\|\mathbf{H}\|_{L^{2}(\Gamma)}|| \nabla_{\Gamma} \mathbf{X}^{*} \|_{L^{2}(\Gamma)}, \\
\left|\int_{\Gamma} \mathbf{H} \cdot\left(\mathbf{H}-\Pi^{*} \mathbf{H}\right)\right| & \leq \delta^{2}\|\mathbf{H}\|_{L^{2}(\Gamma)}|| \nabla_{\Gamma} \mathbf{H} \|_{L^{2}(\Gamma)}, \\
\left|\int_{\Gamma^{*}} \mathbf{H}^{*} \cdot\left(\mathbf{H}-\Pi^{*} \mathbf{H}\right)\right| & \leq \delta^{2}\left\|\mathbf{H}^{*}\right\|_{L^{2}\left(\Gamma^{*}\right)}\left\|\nabla_{\Gamma^{*}} \mathbf{H}\right\|_{L^{2}\left(\Gamma^{*}\right)} .
\end{aligned}
$$

We stress that (4.6) is a condition on the finite element spaces $\mathbb{V}(\Gamma)$ and $\mathbb{V}\left(\Gamma^{*}\right)$ rather than on $\mathbf{X}, \mathbf{X}^{*}, \mathbf{H}$, and $\mathbf{H}^{*}$. Stronger conditions read

$$
\begin{aligned}
\left\|\boldsymbol{\Phi}-\Pi^{*} \boldsymbol{\Phi}\right\|_{L^{2}(\Gamma)} & \leq \delta^{2}\left\|\nabla_{\Gamma} \boldsymbol{\Phi}\right\|_{L^{2}(\Gamma)}, & \forall \boldsymbol{\Phi} \in \mathbb{V}(\Gamma) \\
\left\|\boldsymbol{\Phi}^{*}-\Pi \boldsymbol{\Phi}^{*}\right\|_{L^{2}(\Gamma)} & \leq \delta^{2}\left\|\nabla_{\Gamma} \boldsymbol{\Phi}^{*}\right\|_{L^{2}(\Gamma)}, & \forall \boldsymbol{\Phi}^{*} \in \mathbb{V}(\Gamma) .
\end{aligned}
$$

We prefer condition (4.6) because of being less restrictive and easier to check. 
4.4. The Main Result. We are now in the position to state and prove our main result: our GCAP scheme preserves the accuracy built into the input triple $(\Gamma, \mathbf{X}, \mathbf{H})$.

THEOREM 4.4 (Accuracy of GCAP algorithm). Let $\Gamma$ be a piecewise polynomial approximation of $\gamma$ of degree $k \geq 1$ such that $(\Gamma, \boldsymbol{X}, \boldsymbol{H})$ is a geometrically consistent triple. Let $\left(\Gamma^{*}, \boldsymbol{X}^{*}, \boldsymbol{H}^{*}\right)$ be the geometrically consistent triple obtained from Algorithm 1. Let $\epsilon, \delta>0$ be parameters such that the mesh modification $\boldsymbol{m}: \Gamma \rightarrow \Gamma^{*}$ satisfies assumption (4.5) and the interpolation operators $\Pi, \Pi^{*}$ satisfy (4.6). Let $\boldsymbol{x}$ and $\boldsymbol{h}$ be the identity and total vector curvature of $\gamma$ respectively. Then,

$$
\left.||\left|\nabla_{\gamma}(\boldsymbol{x}-\boldsymbol{X})\left\|_{L^{2}(\gamma)}-\right\| \nabla_{\gamma}\left(\boldsymbol{x}-\boldsymbol{X}^{*}\right)\right|_{L^{2}(\gamma)}|\leq C(\epsilon+\delta) \| \boldsymbol{H}|\right|_{L^{2}(\gamma)}
$$

and

$$
\left|\|\boldsymbol{h}-\boldsymbol{H}\|_{L^{2}(\gamma)}-\left\|\boldsymbol{h}-\boldsymbol{H}^{*}\right\|_{L^{2}(\gamma)}\right| \leq C(\epsilon+\delta)\|\boldsymbol{H}\|_{H^{1}(\gamma)},
$$

where the constant $C>0$ depends only on $d, \epsilon_{0}$, and $\mathcal{L}$, the lift from $\Gamma$ to $\gamma$.

Proof. Let $C>0$ be a generic constant depending only on $d$ and $\mathcal{L}$. We start with the estimate (4.7) for the position. The triangle inequality and Lemma 4.3 yield

$$
\begin{aligned}
|| \mid \nabla_{\gamma}\left(\mathbf{x}-\mathbf{X}^{*}\right) \|_{L^{2}(\gamma)} & -\left\|\nabla_{\gamma}(\mathbf{x}-\mathbf{X})\right\|_{L^{2}(\gamma)} \mid \\
& \leq\left\|\nabla_{\gamma}\left(\mathbf{X}-\mathbf{X}^{*}\right)\right\|_{L^{2}(\gamma)} \leq C|| \nabla_{\Gamma}\left(\mathbf{X}-\mathbf{X}^{*}\right) \|_{L^{2}(\Gamma)}
\end{aligned}
$$

consequently it remains to show that

$$
\left\|\nabla_{\Gamma}\left(\mathbf{X}-\mathbf{X}^{*}\right)\right\|_{L^{2}(\gamma)} \leq C(\epsilon+\delta) \|\left.\mathbf{H}\right|_{L^{2}(\gamma)} .
$$

The definitions of elliptic projection operators $\Pi, \Pi^{*}$ imply

$$
\begin{gathered}
\left\|\nabla_{\Gamma}\left(\mathbf{X}-\mathbf{X}^{*}\right)\right\|_{L^{2}(\Gamma)}^{2} \leq\left|\int_{\Gamma} \nabla_{\Gamma} \mathbf{X}: \nabla_{\Gamma}\left(\mathbf{X}-\Pi \mathbf{X}^{*}\right)-\int_{\Gamma^{*}} \nabla_{\Gamma^{*}} \mathbf{X}^{*}: \nabla_{\Gamma^{*}}\left(\Pi^{*} \mathbf{X}-\mathbf{X}^{*}\right)\right| \\
+\left|\int_{\Gamma^{*}} \nabla_{\Gamma^{*}} \mathbf{X}^{*}: \nabla_{\Gamma^{*}}\left(\mathbf{X}-\mathbf{X}^{*}\right)-\int_{\Gamma} \nabla_{\Gamma} \mathbf{X}^{*}: \nabla_{\Gamma}\left(\mathbf{X}-\mathbf{X}^{*}\right)\right|=: A+B .
\end{gathered}
$$

Lemma 4.2 yields a bound for term $B$

$$
B \leq C \epsilon|| \nabla_{\Gamma} \mathbf{X}^{*}\left\|_{L^{2}(\Gamma)}\right\| \nabla_{\Gamma}\left(\mathbf{X}-\mathbf{X}^{*}\right) \|_{L^{2}(\Gamma)} .
$$

To deal with term $A$, we recall that the triples $(\Gamma, \mathbf{X}, \mathbf{H})$ and $\left(\Gamma^{*}, \mathbf{X}^{*}, \mathbf{H}^{*}\right)$ are $\mathrm{GC}$, the former by assumption and the latter because of (3.4). Hence, we can write

$$
A=\left|\int_{\Gamma} \mathbf{H} \cdot\left(\mathbf{X}-\Pi \mathbf{X}^{*}\right)-\int_{\Gamma^{*}} \mathbf{H}^{*} \cdot\left(\Pi^{*} \mathbf{X}-\mathbf{X}^{*}\right)\right| .
$$

Invoking the definition (3.3) of $\mathbf{H}^{*}$ we see that

$$
\int_{\Gamma^{*}} \mathbf{H}^{*} \cdot\left(\Pi^{*} \mathbf{X}-\mathbf{X}^{*}\right)=\int_{\Gamma} \mathbf{H} \cdot\left(\Pi^{*} \mathbf{X}-\mathbf{X}^{*}\right),
$$

whence term $A$ becomes

$$
A=\left|\int_{\Gamma} \mathbf{H} \cdot\left(\mathbf{X}-\Pi^{*} \mathbf{X}\right)+\mathbf{H} \cdot\left(\mathbf{X}^{*}-\Pi \mathbf{X}^{*}\right)\right| .
$$


Consequently, assumption (4.6) gives

$$
A \leq C \delta^{2}\|\mathbf{H}\|_{L^{2}(\Gamma)}\left(\left\|\nabla_{\Gamma} \mathbf{X}\right\|_{L^{2}(\Gamma)}+\left\|\nabla_{\Gamma} \mathbf{X}^{*}\right\|_{L^{2}(\Gamma)}\right) .
$$

Using the definitions of $\mathbf{X}, \mathbf{X}^{*}, \mathbf{H}^{*}$, in conjunction with Lemma 4.3, we easily deduce

$$
\left\|\nabla_{\Gamma} \mathbf{X}\right\|_{L^{2}(\Gamma)}+\left\|\nabla_{\Gamma} \mathbf{X}^{*}\right\|_{L^{2}(\Gamma)} \leq C\|\mathbf{H}\|_{L^{2}(\gamma)}
$$

and collecting the estimates for terms $A$ and $B$ we arrive at

$$
\left\|\nabla_{\Gamma}\left(\mathbf{X}-\mathbf{X}^{*}\right)\right\|_{L^{2}(\Gamma)}^{2} \leq C \delta^{2}\|\mathbf{H}\|_{L^{2}(\gamma)}^{2}+C \epsilon\|\mathbf{H}\|_{L^{2}(\gamma)}\left\|\nabla_{\Gamma}\left(\mathbf{X}-\mathbf{X}^{*}\right)\right\|_{L^{2}(\Gamma)} .
$$

Therefore, Young inequality yields the desired estimate (4.7).

To prove the estimate (4.8) for curvature, we use again the triangle inequality and Lemma 4.3

$$
\left.||\left|\mathbf{h}-\mathbf{H}^{*}\right|\right|_{L^{2}(\gamma)}-\left\|\mathbf{h}-\mathbf{H}||_{L^{2}(\gamma)}\left|\leq\left\|\mathbf{H}-\mathbf{H}^{*}\right\|_{L^{2}(\gamma)} \leq C\right| \mid \mathbf{H}-\mathbf{H}^{*}\right\|_{L^{2}(\Gamma)},
$$

and rewrite the right-hand side as follows

$$
\begin{aligned}
\left\|\mathbf{H}-\mathbf{H}^{*}\right\|_{L^{2}(\Gamma)}^{2} & \leq\left|\int_{\Gamma} \mathbf{H} \cdot\left(\mathbf{H}-\mathbf{H}^{*}\right)-\int_{\Gamma^{*}} \mathbf{H}^{*} \cdot\left(\mathbf{H}-\mathbf{H}^{*}\right)\right| \\
& +\left|\int_{\Gamma^{*}} \mathbf{H}^{*} \cdot\left(\mathbf{H}-\mathbf{H}^{*}\right)-\int_{\Gamma} \mathbf{H}^{*} \cdot\left(\mathbf{H}-\mathbf{H}^{*}\right)\right|=: D+E .
\end{aligned}
$$

We invoke Lemma 4.2 to bound term $E$

$$
E \leq C \epsilon\left\|\mathbf{H}^{*}\right\|_{L^{2}(\Gamma)}\left\|\mathbf{H}-\mathbf{H}^{*}\right\|_{L^{2}(\Gamma)} .
$$

To deal with $D$ we make use of the definition of $\mathbf{H}^{*}$. To this end, we first split $\mathbf{H}$ as $\mathbf{H}=\Pi^{*} \mathbf{H}+\left(\mathbf{H}-\Pi^{*} \mathbf{H}\right)$ and next rewrite $D$ as

$$
D=\left|\int_{\Gamma} \mathbf{H} \cdot\left(\mathbf{H}-\Pi^{*} \mathbf{H}\right)-\int_{\Gamma^{*}} \mathbf{H}^{*} \cdot\left(\mathbf{H}-\Pi^{*} \mathbf{H}\right)\right| .
$$

Applying (4.6) we infer that

$$
D \leq C \delta^{2}\|\mathbf{H}\|_{H^{1}(\gamma)}^{2}
$$

because, as a consequence of (4.4), the definition (3.3) of $\mathbf{H}^{*}$, and Lemma 4.3,

$$
\left\|\mathbf{H}^{*}\right\|_{L^{2}(\Gamma)}+\left\|\nabla_{\Gamma} \mathbf{H}\right\|_{L^{2}(\Gamma)}+\left\|\mathbf{H}^{*}\right\|_{L^{2}\left(\Gamma^{*}\right)}+\left\|\nabla_{\Gamma^{*}} \mathbf{H}\right\|_{L^{2}\left(\Gamma^{*}\right)} \leq C|| \mathbf{H} \|_{H^{1}(\gamma)} .
$$

Collecting the estimates for $D$ and $E$ we obtain

$$
\left\|\mathbf{H}-\mathbf{H}^{*}\right\|_{L^{2}(\Gamma)}^{2} \leq C \epsilon\left\|\mathbf{H}^{*}\right\|_{L^{2}(\gamma)}\left\|\mathbf{H}-\mathbf{H}^{*}\right\|_{L^{2}(\Gamma)}+C \delta^{2}\|\mathbf{H}\|_{H^{1}(\gamma)}^{2},
$$

and the asserted estimate (4.8) follows from the Young inequality. $\square$

REMARK 4.5 ( $L^{2}$-assumption on curvature). If instead of the last two inequalities of assumption (4.6) we assume that

$$
\left|\int_{\Gamma} \boldsymbol{H}^{2}-\int_{\Gamma^{*}} \boldsymbol{H}^{* 2}\right| \leq \delta^{2} \int_{\Gamma} \boldsymbol{H}^{2}
$$


then (4.8) gets replaced by

$$
\left|\|\boldsymbol{h}-\boldsymbol{H}\|_{L^{2}(\gamma)}-\left\|\boldsymbol{h}-\boldsymbol{H}^{*}\right\|_{L^{2}(\gamma)}\right| \leq C \delta\|\boldsymbol{H}\|_{L^{2}(\gamma)} .
$$

In fact, from (3.3) we have that $\int_{\Gamma^{*}}\left(\boldsymbol{H}^{*}\right)^{2}=\int_{\Gamma} \boldsymbol{H} \boldsymbol{H}^{*}$, whence

$$
\int_{\Gamma}\left(\boldsymbol{H}-\boldsymbol{H}^{*}\right)^{2}=\int_{\Gamma} \boldsymbol{H}^{2}-\int_{\Gamma^{*}} \boldsymbol{H}^{*^{2}} \leq \delta^{2} \int_{\Gamma} \boldsymbol{H}^{2} .
$$

We thus obtain $\left\|\boldsymbol{H}-\boldsymbol{H}^{*}\right\|_{L^{2}(\Gamma)} \leq C \delta\|\boldsymbol{H}\|_{L^{2}(\gamma)}$, which yields (4.10).

REMARK 4.6 (Relaxation on the GC condition). In view of the proof of Theorem 4.4, the modified triple $\left(\Gamma^{*}, \boldsymbol{X}^{*}, \boldsymbol{H}^{*}\right)$ does not need to be strictly GC but rather

$$
\left|\int_{\Gamma^{*}} \boldsymbol{H}^{*} \cdot\left(\Pi^{*} \boldsymbol{X}-\boldsymbol{X}^{*}\right)-\int_{\Gamma^{*}} \nabla_{\Gamma^{*}} \boldsymbol{X}^{*}: \nabla_{\Gamma^{*}}\left(\Pi^{*} \boldsymbol{X}-\boldsymbol{X}^{*}\right)\right| \leq \eta^{2}\left\|\boldsymbol{H}^{*}\right\|_{L^{2}\left(\Gamma^{*}\right)}^{2}
$$

for some constant $\eta$. In this case, (4.7) changes as follows

$$
||\left|\nabla_{\gamma}(\boldsymbol{x}-\boldsymbol{X})\right|_{L^{2}(\gamma)}-\|\left.\left.\nabla_{\gamma}\left(\boldsymbol{x}-\boldsymbol{X}^{*}\right)\right|_{L^{2}(\gamma)}|\leq C(\epsilon+\delta+\eta)||\boldsymbol{H}|\right|_{L^{2}(\Gamma)} .
$$

Moreover, a similar observation holds to relax the $G C$ property of $(\Gamma, \boldsymbol{X}, \boldsymbol{H})$.

REMARK 4.7 (A posteriori mesh modification strategy). The assumptions (4.5) and (4.6) suggest that parameters $\delta$ and $\epsilon$ can be computed a posteriori, namely after performing the mesh modification. This would lead to a strategy to decide whether the mesh modification can be accepted or discarded.

4.5. Basic Mesh Operations. Mesh refinement, coarsening, and smoothing are the three basic mesh operations supported by Algorithm 1 and are executed on $\Gamma$ without reference to the underlying manifold $\gamma$, which we cannot access for geometric flows.

In this section we describe these basic mesh modification procedures, formulate them in the abstract setting of Section 4.2, and study their influence on geometric accuracy. In particular, we show with examples that the standard approach (STD) yields accuracy deterioration, which is not observed when using our geometrically consistent accuracy preserving (GCAP) algorithm.

To explore the adverse effects of mesh modification, we put ourselves in the best possible scenario: we consider a $\mathrm{GC}$ triple $(\Gamma, \mathbf{X}, \mathbf{H})$ for the unit circle or sphere $\gamma$, and investigate refinement, coarsening and mesh smoothing for both piecewise linear and quadratic finite elements. We use the same polynomial degree $k$ for both $\mathbf{X}$ and $\mathbf{H}$, as stated in (2.3), and postpone the study of mismatch until Section 5.4. The GC triples for the experiments are obtained as follows: $\Gamma$ is a quasi-uniform partition of $\gamma$ with nodes lying on $\gamma, \mathbf{H}$ is obtained by interpolating the exact curvature at these nodes and $\mathbf{X}$ is the solution to (2.3).

We measure the errors in the $L^{2}(\Gamma)$ norm, for which we resort to a lift from $\Gamma$ to $\gamma$. In fact, if $\boldsymbol{\Phi} \in \mathbb{V}(\Gamma)$ is an approximation of a function $\varphi: \gamma \rightarrow \mathbb{R}^{d+1}$, then by $L^{2}$-error on $\boldsymbol{\varphi}$ we mean $\|\boldsymbol{\varphi}-\boldsymbol{\Phi}\|_{L^{2}(\Gamma)}$, where $\boldsymbol{\varphi}$ indicates the lift of $\boldsymbol{\varphi}$ onto $\Gamma$.

We show that naive mesh modification yields an $L^{2}$-accuracy for vector curvature $\mathbf{H}$ of suboptimal order $\mathcal{N}^{(k-1) / d}$, whence it gives rise to a decay rate of the $L^{2}$-error of order $O(1)$ for $k=1$. This is caused by explicit computation with $\Delta_{\Gamma}[9,14]$; our GCAP method, instead, restores optimal order. We choose bisection to describe refinement/coarsening, and our own mesh smoothing method, but emphasize that our findings apply to other choices as well. 
4.5.1. Mesh Refinement. A compatible bisection patch is a set of elements sharing the same refinement edge. The atomic refinement operation consists of adding a mid-node on the refinement edge of one such patch. If the patch is incompatible, then recursion is employed which keeps conformity but spreads the effect of refinement. If $\mathcal{T}$ stands for the current mesh on $\Gamma$ and $\mathcal{T}^{*}$ for a conforming refinement, we write $\mathcal{T}^{*} \geq \mathcal{T}$, which is meaningful in the sense of trees, and note that the new manifold $\Gamma^{*}$ coincides with $\Gamma$. We refer to $[1,16,4,24]$ for a discussion on bisections.

To refine an element $K \in \mathcal{T}$, we first bisect the reference element $\widehat{K}$ across the corresponding refinement edge, and next map via $\mathbf{F}_{K}^{k}$ (see Section 3.1) the new Lagrange nodes $\widehat{\mathbf{y}}_{j}^{*} \in \widehat{K}$ of degree $k$, namely $\mathbf{y}_{j}^{*}=\mathbf{F}_{K}^{k}\left(\widehat{\mathbf{y}}_{j}^{*}\right) \in K$. If $K$ belongs to a compatible bisection patch, then bisection remains local. Otherwise, recursion is employed in the search for a bisection patch and there is a spreading effect in the vicinity of $K$. In this case, $\Gamma^{*}=\Gamma, \boldsymbol{m}$ is the identity, the corresponding triangulations satisfy $\mathcal{T} \leq \mathcal{T}^{*}$ in the sense of trees $[4,19]$, and $\mathbb{V}(\Gamma) \subset \mathbb{V}\left(\Gamma^{*}\right)$. This implies

REMARK 4.8 (Refinement). For refinement $\boldsymbol{m}:=i d_{\Gamma}: \Gamma \rightarrow \Gamma^{*} \equiv \Gamma$, so that $\epsilon=0$ and (4.5) as well as (4.9) are trivially satisfied, whence

$$
\begin{aligned}
||\left|\nabla_{\gamma}(\boldsymbol{x}-\boldsymbol{X})\right|_{L^{2}(\gamma)}-\left\|\nabla_{\gamma}\left(\boldsymbol{x}-\boldsymbol{X}^{*}\right)\right\|_{L^{2}(\gamma)} \mid & \leq C \delta\|\boldsymbol{H}\|_{L^{2}(\gamma)}, \\
\left|\|\boldsymbol{h}-\boldsymbol{H}\|_{L^{2}(\gamma)}-\left\|\boldsymbol{h}-\boldsymbol{H}^{*}\right\|_{L^{2}(\gamma)}\right| & =0 .
\end{aligned}
$$

Compare with the adverse effect of STD refinements shown in Section 2.

4.5.2. Mesh Coarsening. Removing a node is a process dual to refinement. The atomic coarsening operation is the removal of the central node of a compatible bisection patch. This can only happen if all elements in the patch are marked for coarsening, so this operation is naturally more conservative than refinement. Once a node $\mathbf{x}_{0}$ of $\mathcal{T}$ is removed, pairs of elements containing $\mathbf{x}_{0}$ are merged into their parent element, which may not occupy the same region in space as they do in the flat case. To do so, we just have to undo the refinement procedure described above upon merging the corresponding elements in the reference element $\widehat{K}$ and mapping the new nodes back to $\Gamma$. This procedure is illustrated in Figure 4.1 for linear elements on

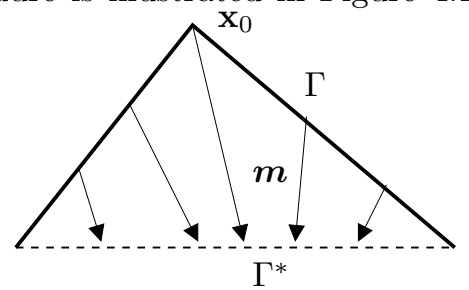

FIG. 4.1. Coarsening procedure for $k=1$ (linear elements) and $d=1$ (curve). The curve $\Gamma^{*}$ is obtained from $\Gamma$ by removing the node $x_{0}$.

curves $(k=d=1)$. Note that the mesh modification $\boldsymbol{m}$ can be characterized by

$$
\left.\boldsymbol{m}\right|_{K}=\left[I_{\mathcal{T}^{*}}^{k}\left(\mathbf{F}_{K}^{k}\right)\right] \circ\left(\mathbf{F}_{K}^{k}\right)^{-1},
$$

where $I_{\mathcal{T}^{*}}^{k}$ is the nodal interpolant of degree $k$ with respect to the new subdivision $\mathcal{T}^{*}$ and $\mathbf{F}_{\bar{K}}^{k}$ is the polynomial map of degree $\leq k$ defined in Section 3.1. If $\mathbf{x}_{0}^{*} \in \Gamma^{*}$ is the image of $\widehat{\mathbf{x}}_{0} \in \widehat{K}$, we realize that $\mathbf{x}_{0}^{*} \notin \Gamma$ for linear elements, $\mathbf{x}_{0}^{*} \in \Gamma$ for quadratic elements, and always $\Gamma^{*} \neq \Gamma$.

REMARK 4.9 (Sensitivity of $\delta$ to coarsening and refinement). Condition (4.6) can be written as a sum of element contributions and implies that where $\boldsymbol{H}$ is small, performing refinement or coarsening does not significantly affect the value of $\delta$. 
In light of (2.4) and the fact that the nodes of $\Gamma^{*}$ belong to $\Gamma$, for curves we realize that the resulting triple $\left(\Gamma^{*}, \mathbf{X}^{*}, \mathbf{H}^{*}\right)$ would benefit from a super-approximation property. Therefore, to illustrate the deterioration of accuracy for coarsening, we must resort to a 2D surface as depicted in Figure 4.2. This figure shows a quasi-uniform

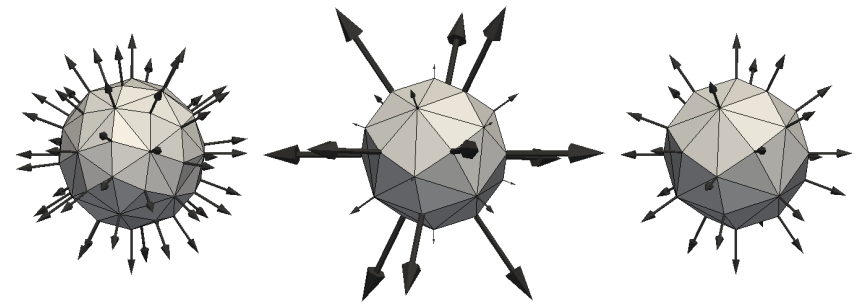

FIG. 4.2. Quasi-uniform polyhedral surface $\Gamma$ interpolating the unit sphere $\gamma$, and GC triple $(\Gamma, \boldsymbol{X}, \boldsymbol{H})$ with $\boldsymbol{H} \approx 2 \boldsymbol{\nu}$ (left). Uniform coarsening $\Gamma^{*}$ and computation on $\left(\boldsymbol{X}^{*}, \boldsymbol{H}^{*}\right)$ satisfying (2.3) via the STD method (center) and our GCAP method (right). STD chooses $\boldsymbol{X}^{*}$ to be the interpolant of $\boldsymbol{X}$ and $\left(\Gamma^{*}, \boldsymbol{X}^{*}, \boldsymbol{H}^{*}\right)$ suffers from a loss of accuracy. Our GCAP method yields a GC triple and preserves accuracy, but $\boldsymbol{X}^{*}$ is not the interpolant of $\boldsymbol{X}$. The magnitude of all the curvature vectors are scaled down by a factor of 0.3 .

piecewise linear surface $\Gamma$ interpolating the unit sphere, and a $\mathrm{GC}$ triple $(\Gamma, \mathbf{X}, \mathbf{H})$ with $\mathbf{H} \approx 2 \boldsymbol{\nu}$ (left). We next coarsen $\Gamma$ uniformly to obtain $\Gamma^{*}$, and apply both the standard (STD) coarsening method (center) and our GCAP method (right). We realize that the STD method gives a vector curvature $\mathbf{H}^{*}$ with wrong magnitude while the GCAP method preserves its accuracy. To document that this finding is not coincidental and cannot be fixed by increasing resolution, we compute a sequence of uniform refinements and corresponding uniform coarsenings on $\gamma$, and report the results in Figure 4.3. For the STD method we observe lack of convergence order $\mathcal{N}^{0}$ for linears and order $\mathcal{N}^{-1 / 2}$ for quadratics, whereas our GCAP method restores the optimal rates $\mathcal{N}^{-1}$ and $\mathcal{N}^{-3 / 2}$ typical of dimension $d=2$ in terms of degrees of freedom $\mathcal{N}$.
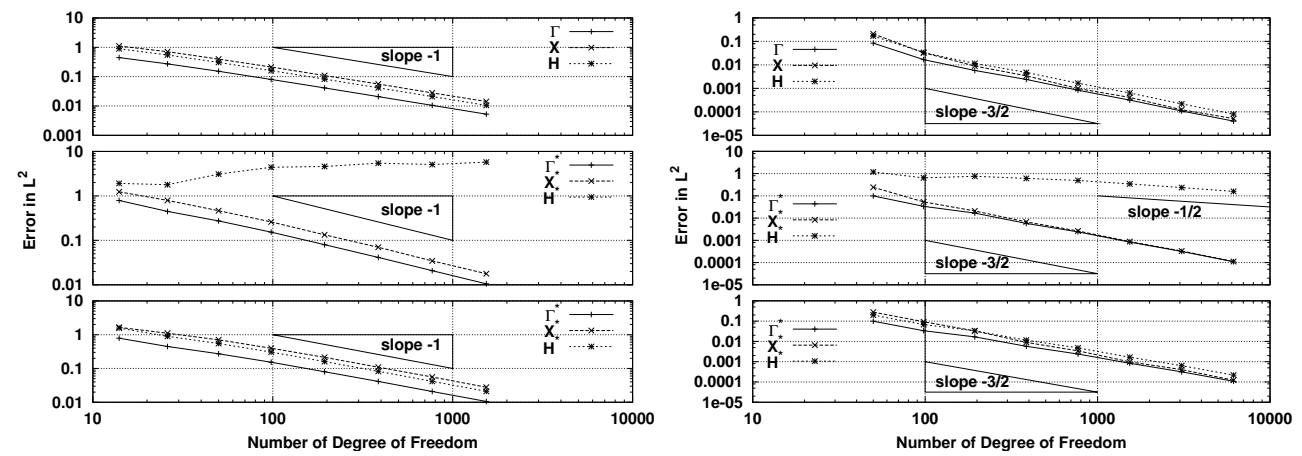

FIG. 4.3. Effect of one global coarsening on a sequence of piecewise linear (left column) and quadratics (right column) approximation of the unit sphere with decreasing meshsize. Each plot shows the $L^{2}$ error decay for the parametrization of the surface $\Gamma$, the curvature $\boldsymbol{H}$ and the position $\boldsymbol{X}$ vs the number of degrees of freedom $\mathcal{N}$ before the modification. The rows correspond to the GC triplet initially (top), after standard coarsening (STD) (middle) and after the GCAP method (bottom). The decay of the $L^{2}$-errors using STD for linears $k=1$ is $\mathcal{N}^{0}$ (no decrease) whereas that for quadratics $k=2$ is suboptimal $\mathcal{N}^{-1 / 2}$. In contrast, our GCAP method restores optimal orders $\mathcal{N}^{-1}$ and $\mathcal{N}^{-3 / 2}$. The initial triplet is obtained by interpolating $\Gamma$ and $\boldsymbol{H}$ and then solving (2.2) for $\boldsymbol{X}$. 
4.5.3. Mesh Smoothing. The third type of mesh modification consists of moving the position of the nodes describing $\Gamma$. Mesh smoothing is an important instance of this type of modification. The latter is crucial to keep mesh regularity, especially under large domain deformations which distort the mesh. It is thus significant to include mesh quality control as part of the design of a robust method. Here we just experiment with our algorithm of [20], whose atomic mesh smoothing operation is carried out on stars. It is a hybrid affine-quadratic approach for isoparametric surfaces, which simply relocates the center node of a star to improve its quality, and then adjusts the quadratic surplus. More precisely, in order to determine the new position of a given vertex $\mathbf{x}$ we proceed as follows and refer to Figure 4.4. Let $\bar{\omega}_{\mathbf{x}}$ be the linear star of center $\mathbf{x}$ and vertices $\left\{\mathbf{x}_{i}\right\}$ with the convention that $\mathbf{x}_{0}=\mathbf{x}$. We consider a plane $\pi_{\mathbf{x}}$ approximating $\bar{\omega}_{\mathbf{x}}$ and denote by $\left\{\mathbf{z}_{i}\right\}$ the projections into $\pi_{\mathbf{x}}$ (along the normal direction to $\pi_{\mathbf{x}}$ ) of all $\left\{\mathbf{x}_{i}\right\}$ of $\bar{\omega}_{\mathbf{x}}$. We next perform a smoothing procedure on $\pi_{\mathbf{x}}$ leading to $\mathbf{z}_{0}^{*}$, the new location on $\pi_{\mathbf{x}}$ of the node $\mathbf{z}_{0}$. The new location $\mathbf{x}^{*}$ of $\mathbf{x}$ on $\Gamma$ is obtained by lifting back $\mathbf{z}^{*}$ to $\Gamma$ (along the normal direction to $\pi_{\mathbf{x}}$ again), which gives rise to a linear star $\bar{\omega}_{\mathbf{x}^{*}}$ with better shape regularity than $\bar{\omega}_{\mathbf{x}}$. The higher order star $\omega_{\mathbf{x}^{*}}$ results from isoparametric interpolation of the nodes $\left\{\mathbf{x}_{i}\right\} \backslash\{\mathbf{x}\} \cup\left\{\mathbf{x}^{*}\right\}$ in $\omega_{\mathbf{x}}$. This procedure induces a mesh modification $\boldsymbol{m}_{i}$ for each vertex $\mathbf{x}_{i}$ of $\mathcal{T}$, and their concatenation defines the global mesh modification $\boldsymbol{m}$ that changes the piecewise polynomial approximation $\Gamma$ of $\gamma$ of degree $k \geq 1$ into another one $\Gamma^{*} \neq \Gamma$ with the same degree. Details about how to obtain $\pi_{\mathbf{x}}$, measure element quality, or perform the star optimization procedure on $\pi_{\mathbf{x}}$ are irrelevant for the present discussion.

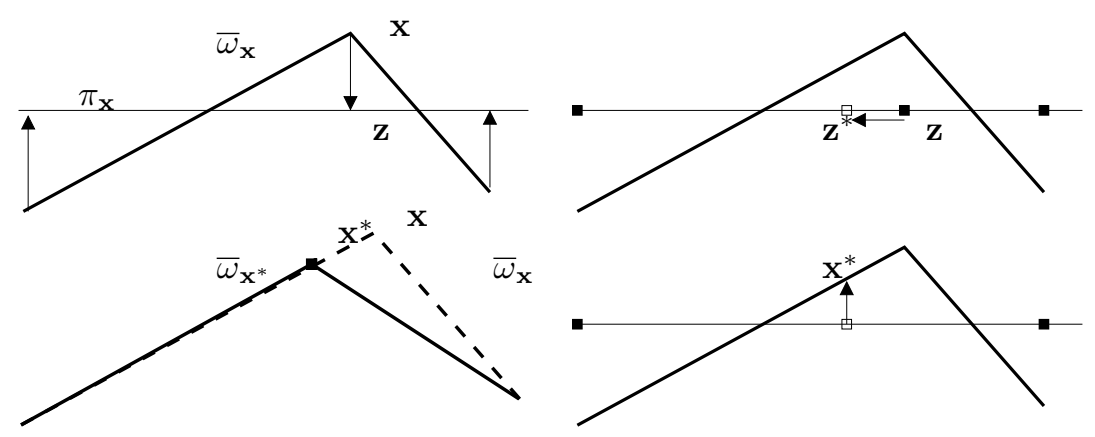

FIG. 4.4. (Clockwise) Smoothing procedure of the linear star when $d=1$ (curve). Top left: the nodes $\boldsymbol{x}_{i}\left(\boldsymbol{x}:=\boldsymbol{x}_{0}\right)$ are projected into the plane $\pi_{\boldsymbol{x}}$. Top right: the center node $\boldsymbol{z}$ corresponding to the projection of $\boldsymbol{x}$ is moved in a more suitable position $\boldsymbol{z}^{*}$. Bottom right: the new position $\boldsymbol{x}^{*}$ is determined by projecting back the node to the original curve. Bottom left: the smoothed out linear star $\bar{\omega}_{x^{*}}$ is obtained by linear interpolation.

The new nodes are relocated on $\Gamma$ using the local finite element map and reconnected via isoparametric interpolation. This leads to $\Gamma^{*} \neq \Gamma$ for any polynomial degree $k$.

We would like to remark that computing the global mesh modification $\boldsymbol{m}$, and thus $\mathbf{H}^{*}$ in (3.3), can be tedious in practice. The accuracy of the GC Algorithm will be preserved if instead of $\mathbf{H}^{*}$ an approximation of the same order $\widetilde{\mathbf{H}}^{*}$ is used. We provide now a simple procedure to construct such $\widetilde{\mathbf{H}}^{*}$ that hinges on isoparametric interpolation of $\mathbf{H}$. Mesh smoothing gives rise to new positions of the nodes in $\Gamma$, and $\Gamma^{*}$ results from local isoparametric interpolation of these nodes. We thus set $\widetilde{\mathbf{H}}^{*}$ to be the interpolant of degree $k$ related to the values of $\mathbf{H}$ at these new locations, computed recursively on stars. For an underlying exact surface $\gamma \in C^{k+3}$, we expect 
from interpolation theory that

$$
\left\|\mathbf{H}^{*}-\widetilde{\mathbf{H}}^{*}\right\|_{L^{2}\left(\Gamma^{*}\right)}=O\left(h^{k+1}\right),
$$

thereby justifying the use of $\widetilde{\mathbf{H}}^{*}$ instead of $\mathbf{H}^{*}$.
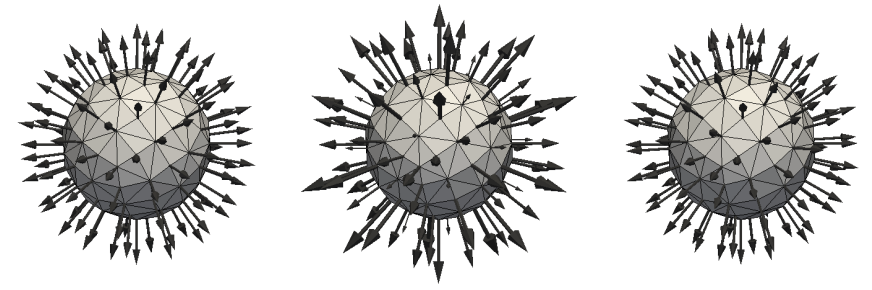

FIG. 4.5. Quasi-uniform polyhedral surface $\Gamma$ interpolating the unit sphere $\gamma$, and $G C$ triple $(\Gamma, \boldsymbol{X}, \boldsymbol{H})$ with $\boldsymbol{H} \approx 2 \boldsymbol{\nu}$ (left). Mesh smoothing gives $\Gamma^{*}$, with $\left(\boldsymbol{X}^{*}, \boldsymbol{H}^{*}\right)$ satisfying (2.3) via the STD method (center) and our GCAP method (right). STD chooses $\boldsymbol{X}^{*}$ to be the interpolant of $\boldsymbol{X}$ and exhibits loss of accuracy (see Figure 4.6). The magnitude of all the curvature vectors are scaled down by a factor of 0.3 .
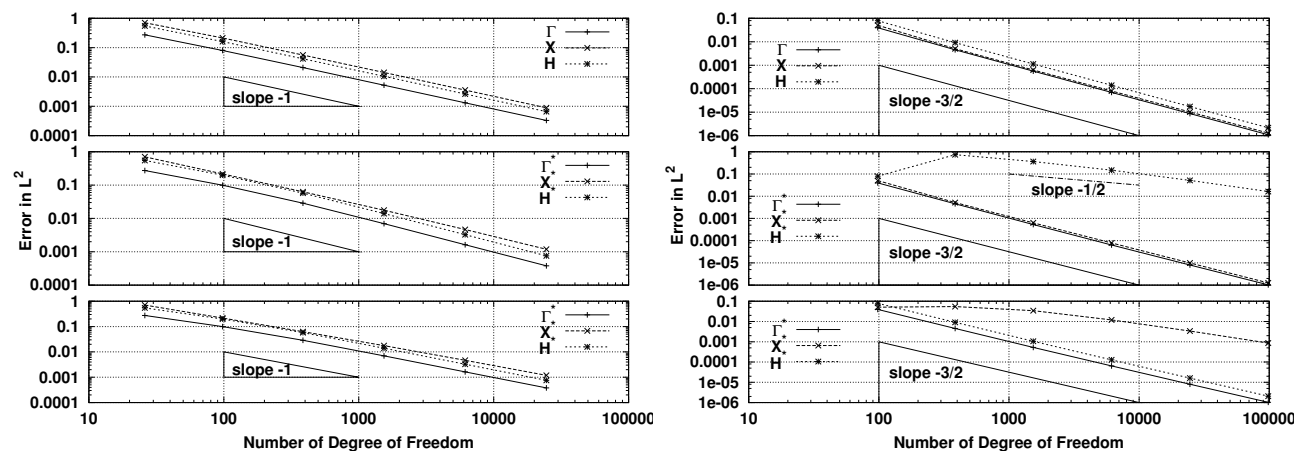

FIG. 4.6. Effect of our smoothing procedure on a sequence of piecewise linear (left column) and quadratics (right column) approximation of the unit sphere with decreasing meshsize. On each column, the $L^{2}$ error on the surface $\Gamma$, the curvature $\boldsymbol{H}$ and the position $\boldsymbol{X}$ are plotted versus the number of degrees of freedom before the smoothing (top), after the smoothing using a standard procedure to reallocate the position of the nodes (middle) and our GCAP smoothing method (bottom). The decay rate of the $L^{2}$-errors for STD using linears is less that $1 / 2$ whereas using quadratics is 1. In contrast, our GCAP algorithm restores orders $1 / 2$ and $3 / 2$.

Figure 4.5 depicts this mesh modification in the case of a unit sphere. The behavior of the standard smoothing (STD) method, for which $\mathbf{X}^{*}$ is the identity over $\Gamma^{*}$, corresponds to barely visible node reallocation and the lack of accuracy is less dramatic than in Figure 4.2. This cannot be repaired with increasing resolution, as shown in Figure 4.6, in which we report the results for a sequence of uniform refinements followed by mesh smoothing. The convergence rates are similar to those for coarsening.

5. Applications to Geometric Flows. In applications to geometric flows [12, $11,21,2,6,7,5]$ the key for GC lies on the semi-implicit time discretization of $(2.1)$, which leads to the equation

$$
\mathbf{h}^{n+1}=-\Delta_{\gamma^{n}} \mathbf{x}^{n+1} .
$$


We expect $\mathbf{h}^{n+1}$ and $\mathbf{x}^{n+1}$ to be good approximations of $\mathbf{h}\left(t_{n+1}\right)$ and $\mathbf{x}\left(t_{n+1}\right)$, but the geometric constitutive relation (5.1) is not satisfied on $\gamma^{n+1}$ but $\gamma^{n}$; this decoupling is crucial for the triple $\left(\gamma^{n}, \mathbf{x}^{n+1}, \mathbf{h}^{n+1}\right)$ to be GC. The relation between the new position $\mathbf{x}^{n+1}$, velocity $\mathbf{v}^{n+1}$, and the identity $\mathbf{x}^{n}$ over $\gamma^{n}$, reads

$$
\mathbf{x}^{n+1}=\mathbf{x}^{n}+\delta t^{n+1} \mathbf{v}^{n+1},
$$

where $\delta t^{n+1}>0$ is the timestep. We can thus equivalently write (5.1) as follows:

$$
\mathbf{h}^{n+1}=-\Delta_{\gamma^{n}}\left(\mathbf{x}^{n}+\delta t^{n+1} \mathbf{v}^{n+1}\right) .
$$

This shows that the notion of GC implicitly involves the timestep. We explore below the lack of robustness of piecewise linear finite elements via several examples, along with some remedies. Most issues can be cured by piecewise quadratic finite elements, provided we give up one order of accuracy on the decay of the approximation error.

First, the effect of performing standard mesh modifications is examined for the mean curvature and Willmore flows where relation (5.3) is used. Next, we draw two parallels between non-GC modifications and (i) the choice of the finite element spaces to describe $\Gamma$ and $\mathbf{X}$, (ii) the sensitivity to the choice of the time step in (5.2).
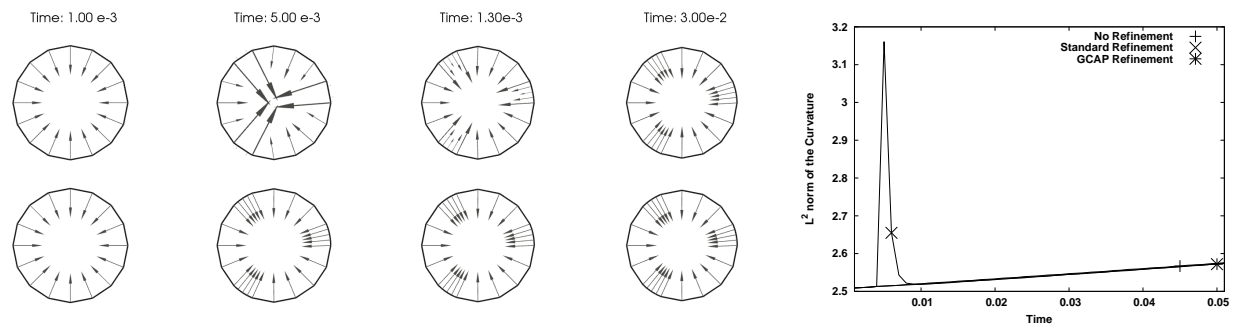

FIG. 5.1. An initial polygonal interpolating the unit circle is evolved with a discrete mean curvature flow using a fixed timestep of $\delta t^{n+1}=10^{-3}$ and (5.4). After 5 time steps 3 elements are refined twice using standard bisection (top) and GCAP refinement (bottom). The pictures show the discrete velocities given by both schemes at different times of the simulation, and the plot provides the $L^{2}$-norm of the curvature vs time. In contrast to the botton row, the length of the arrows is quite not uniform for the top row after 13 time steps, which correspond to a noticeable peak in the plot. The flow recovers after 30 time steps: the arrows become visually even and the plots for standard bisection and GCAP refinement concide.

5.1. Mesh Refinement for Mean Curvature Flow. This flow is governed by the 2 nd order PDE: $\mathbf{v}=-\mathbf{h}$. In view of (5.3), its discrete version on $\Gamma^{n}$ reads

$$
\int_{\Gamma^{n}} \mathbf{V}^{n+1} \boldsymbol{\Phi}+\delta t^{n+1} \nabla_{\Gamma^{n}} \mathbf{V}^{n+1}: \nabla_{\Gamma^{n}} \boldsymbol{\Phi}=-\int_{\Gamma^{n}} \nabla_{\Gamma^{n}} \mathbf{X}^{n}: \nabla_{\Gamma^{n}} \boldsymbol{\Phi},
$$

where $\mathbf{X}^{n}$ is the identity on $\Gamma^{n}$. In Figure 5.1, we illustrate the effect of standard refinement on the flow of a circle (top row), along with our GCAP refinement (botton row). This perturbation is noticeable but not strong enough to change the flow, which recovers after 30 iterations and gives the same evolution as with GCAP refinement.

5.2. Mesh Refinement for Willmore Flow. For surfaces of dimension $d=2$, this flow is governed by the 4 th order $\operatorname{PDE}[3,5,6,7,12,21]$

$$
v=\Delta_{\gamma} h+\frac{1}{2} h^{3}-2 h \kappa \quad(d=2) ; \quad v=\Delta_{\gamma} h+\frac{1}{2} h^{3} \quad(d=1),
$$


which relates the normal velocity $v$, scalar total curvature $h$, and Gauss curvature $\kappa$. We examine the effect of mesh refinement on the dimension-independent vector equation derived in [5]

$$
\begin{aligned}
& \int_{\Gamma^{n}} \mathbf{V}^{n+1} \boldsymbol{\Phi}+\nabla_{\Gamma^{n}} \mathbf{H}^{n+1}: \nabla_{\Gamma^{n}} \boldsymbol{\Phi} \\
& \quad-\left(\nabla_{\Gamma^{n}} \mathbf{X}^{n}+\left(\nabla_{\Gamma^{n}} \mathbf{X}^{n}\right)^{T}\right) \nabla_{\Gamma^{n}} \mathbf{H}^{n+1}: \nabla_{\Gamma^{n}} \boldsymbol{\Phi}+\frac{1}{2} \operatorname{div}_{\Gamma^{n}} \mathbf{H}^{n+1} \operatorname{div}_{\Gamma^{n}} \boldsymbol{\Phi}=0,
\end{aligned}
$$

for all $\mathbf{\Phi} \in \mathbb{V}\left(\Gamma^{n}\right)$ piecewise linear (see Figure 5.2); here $\mathbf{X}^{n}$ is the identity of $\Gamma^{n}$. We stress that this effect is similar for the alternative methods of Dziuk [12] and Rusu [21] and point out that performing standard refinements changes the flow and thus yields a different, and so incorrect, evolution. The discrepancy appears to be proportional to the meshsize, so repeated refinement might have a devastating accuracy effect.
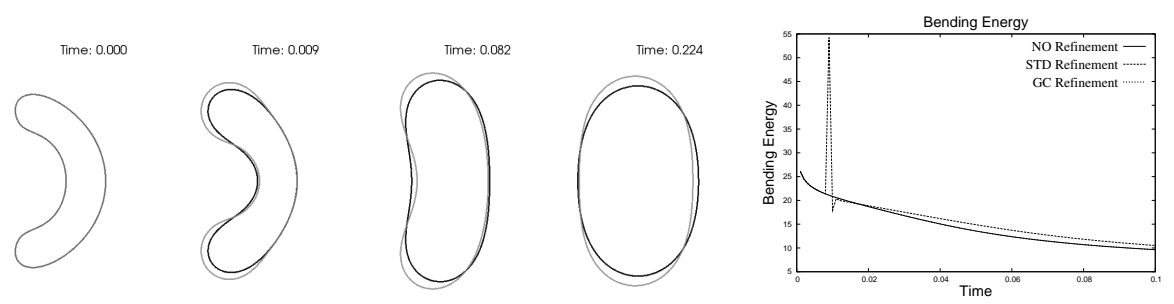

FIG. 5.2. Two dimensional Willmore flow for an initial boomerang shape using the discrete scheme (5.6) for piecewise linear elements and fixed timestep of $10^{-3}$. After 8 time steps, we perform a global standard bisection of each segment. The curves for $G C$ refinement and no refinement are on top of each other (black), whereas that for standard refinement (gray) is not. This striking effect of refinement changes the flow and is rather tricky to detect. In the graph of Willmore (or bending) energy vs time, we depict 3 curves: the GCAP method curve coincides with the one without refinement, whereas the standard refinement exhibits an artificial oscillatory peak of the energy and different asymptotics.

5.3. GC Initial Triple for Geometric Flows. Initializing discrete schemes for geometric flows requires a discretization of $\gamma(0)$ and sometimes also of $\mathbf{h}(0)$. A GC triple with a non-optimal decay rate for the geometric error on $\mathbf{x}$ and $\mathbf{h}$ may distort the flow (see Figure 5.3 top row), or require so small timesteps to avoid node crossing that the flow would barely evolve; this has already been reported $[3,5,12,21]$. Using piecewise quadratics $(k=2)$ gives rise to GC triples $[2,5,14]$ with a suboptimal geometric error decay, which happens to be the optimal for linears.

The key question is to develop a practical method for $k=1$. We propose a simple method based on quadratics. Let $\Gamma_{2}^{0}$ be a quadratic interpolant of $\gamma(0)$ and $\Gamma_{1}^{0}$ the linear interpolant of $\gamma(0)$ obtained by ignoring the quadratic nodes of $\Gamma_{2}^{0}$. If $\mathbf{X}_{2}^{0}$ is the identity over $\Gamma_{2}^{0}$, and $\mathbf{H}_{2}^{0}$ is computed explicitly according to (2.3), then the triple $\left(\Gamma_{2}^{0}, \mathbf{X}_{2}^{0}, \mathbf{H}_{2}^{0}\right)$ is GC. We next restrict $\mathbf{H}_{2}^{0}$ to the linear nodes (or vertices of $\Gamma^{0}$ ), thereby getting $\mathbf{H}_{1}^{0}$, and solve for a piecewise linear $\mathbf{X}_{1}^{0}$ over the piecewise linear interpolant $\Gamma_{1}^{0}$ of $\gamma(0)$. Since $\mathbf{H}_{2}^{0}$ possesses first order accuracy, then the triple $\left(\Gamma_{1}^{0}, \mathbf{X}_{1}^{0}, \mathbf{H}_{1}^{0}\right)$ is GC with the same accuracy, but $\mathbf{X}_{1}^{0}$ is not the identity on $\Gamma_{1}^{0}$. The botton row of Figure 5.3 corroborates this assertion for the mean curvature flow of the unit sphere.

5.4. Mismatch of Finite Element Spaces. Following Bänsch [2], we consider the time-dependent Stokes system on a bounded deformable domain $\Omega(t)$ with surface 


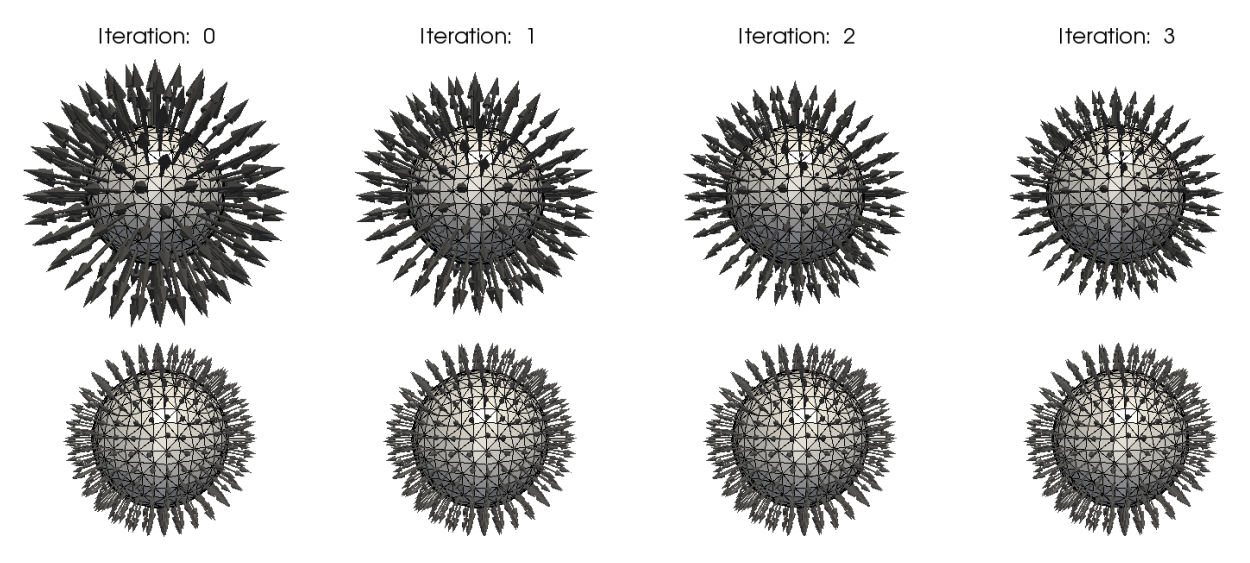

FIG. 5.3. Comparison of the mean curvature flow of the unit sphere $\gamma(0)$ with uniform timestep $10^{-3}$ and polynomial degree $k=1$. The top row shows the triple $\left(\Gamma^{0}, \boldsymbol{X}^{0}, \boldsymbol{H}^{0}\right)$ obtained by interpolation of $\gamma(0)$. The botton row depicts our GCAP triple. The arrows represent vector curvature and are scaled by 0.3 . The arrows in the top row recover their uniformity and correct magnitude only after 20 time iterations (or steps).

tension on $\gamma(t)=\partial \Omega(t)$. Its discrete formulation on an approximate domain $\Omega^{n}$ with boundary $\Gamma^{n}$ reads as follows: we seek Taylor-Hood approximations $\mathbf{V}^{n+1}$ and $P^{n+1}$ of the velocity and pressure at time $t^{n+1}$ such that

$$
\begin{aligned}
\frac{1}{\delta t} \int_{\Omega^{n}}\left(\mathbf{V}^{n+1}-\mathbf{V}^{n}\right) \cdot \boldsymbol{\Phi} & +\int_{\Omega^{n}} \mathbf{T}^{n+1}: \nabla \boldsymbol{\Phi} \\
& +\delta t \int_{\Gamma^{n}} \nabla_{\Gamma^{n}} \mathbf{V}^{n+1}: \nabla_{\Gamma^{n}} \boldsymbol{\Phi}=-\int_{\Gamma^{n}} \nabla_{\Gamma^{n}} \mathbf{X}^{n}: \nabla_{\Gamma^{n}} \mathbf{\Phi},
\end{aligned}
$$

plus the incompressibility condition, where $\mathbf{T}^{n+1}=\nabla \mathbf{V}^{n+1}+\left(\nabla \mathbf{V}^{n+1}\right)^{T}+P^{n+1} \mathbf{I}$. The last two terms in (5.7) expressed the balance of forces at the interface $\Gamma^{n}: \mathbf{T}^{n+1} \boldsymbol{\nu}^{n}=$ $-\mathbf{H}^{n+1}$. The choice of Taylor-Hood elements (piecewise quadratic velocities and linear pressures) is a natural compromise between accuracy and solvability in fluid dynamics. The use of isoparametric finite elments is also natural to capture the full strength of quadratic elements, and is advocated in [2]. We discovered that the use of straight elements does not just reduce the approximation order but instead might lead to lack of convergence. We provide now computational insight. Siebert also encountered similar problems and found an explanation (unpublished work).

We first observe that the essense of this pathological behavior can be captured with the much simpler mean curvature flow (5.4); in fact the presence of a fluid in the bulk alleviates the problem to some extend. We let $\Gamma^{n}, \mathbf{X}^{n}$ be piecewise linears, with $\mathbf{X}^{n}$ being identity over $\Gamma^{n}$, and $\mathbf{V}^{n+1}$ be piecewise quadratic. Having computed $\mathbf{V}^{n+1}$, we obtain the new position $\mathbf{X}^{n+1}$ of the interface $\Gamma^{n+1}$ by adding the piecewise linear interpolant of $\delta t \mathbf{V}^{n+1}$ to $\mathbf{X}^{n}$. We certainly do not expect this discretization to be better than that of piecewise linear elements. However, the striking fact is that it is much worse, as documented in Figure 5.4 for a circle and $d=1$.

To shed light on this behavior, we run a sequence of experiments with decreasing timestep $\delta t$ and constant initial meshsize; see Figure 5.5 (left). We see that any collapsing time between 0.45 and 0.2 is reachable as $\delta t$ decreases whereas the exact one is 0.5 . It is worth observing that for $\delta t \rightarrow 0,(5.4)$ yields a velocity $\mathbf{V}^{n+1}$, and so 

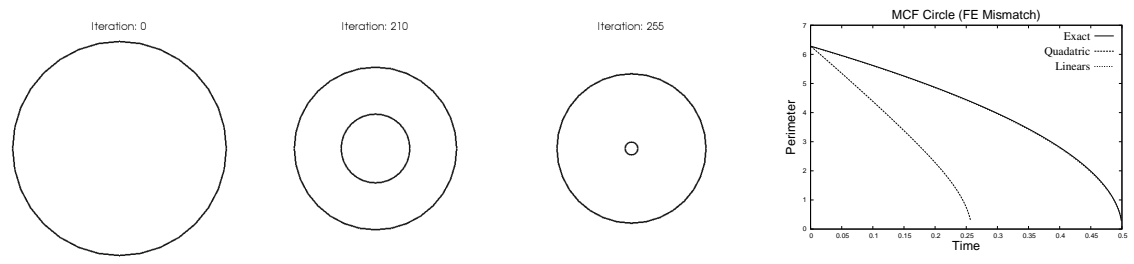

FIG. 5.4. Two mean curvature flows of the unit circle with fixed timestep $10^{-3}$, and piecewise linear approximation $\Gamma^{n}$ of the curve. One scheme uses quadratic elements for velocity whereas the other uses linears. The former yields a larger velocity, with nearly double magnitude, and leads to a collapse to a point way before the exact time $t=0.5$. The plot of perimeter of the approximate curves $\Gamma^{n}$ vs time clearly depicts the two evolutions; the exact perimeter is $p(t)=2 \pi(1-2 t)^{1 / 2}$. We explore the effects of changing meshsize and timesteps in Figure 5.5.

curvature $\mathbf{H}^{n+1}$, by the explicit computation with quadratic finite elements $\mathbb{V}\left(\Gamma^{n}\right)$

$$
\mathbf{H}^{n+1} \in \mathbb{V}\left(\Gamma^{n}\right): \quad \int_{\Gamma^{n}} \mathbf{H}^{n+1} \cdot \mathbf{\Phi}=\int_{\Gamma^{n}} \nabla_{\Gamma^{n}} \mathbf{X}^{n}: \nabla_{\Gamma^{n}} \boldsymbol{\Phi}, \quad \forall \boldsymbol{\Phi} \in \mathbb{V}\left(\Gamma^{n}\right)
$$

For the midpoints the right-hand side of (5.8) gives a zero contribution which, combined with the contribution at the vertices, yields and oscillatory function $\mathbf{H}^{n+1}$. This behavior is similar that the one described already in Figure 2.4, and explains Figure 5.5 (left). In contrast, Figure 5.5 (right) displays asymptotics for the perimeter as the meshsize decreases and the timestep remains fixed. In the limit, we get $\mathbf{V}^{n+1}=-\Delta_{\Gamma^{n}} \mathbf{X}^{n+1}$ which is consistent with the correct flow and explains Figure 5.5 (right). Consequently, we conclude that adequate relations between timestep and meshsize for convergence are rather tricky to select in practice.
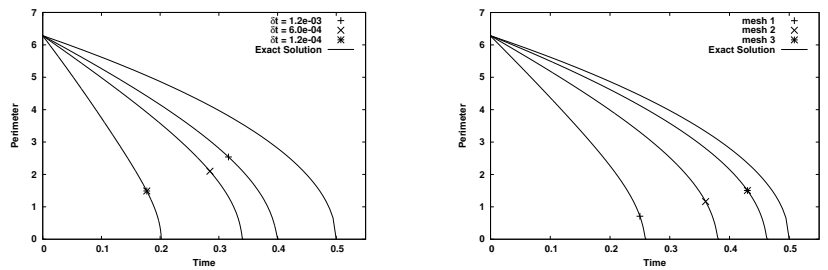

FIG. 5.5. Mean curvature flow of the unit circle The exact formula for the perimeter as a function of time is given by $P(t)=2 \pi \sqrt{1-2 t}$. (Left) Fixed meshsize and three different constant timesteps: $\delta t=1.2 e-3,6.0 e-4,1.2 e-4$. The polynomial mismatch enables us to reach any collapsing time between 0.2 and 0.45 , whereas the exact one is 0.5 . This gives rise to an incorrect dynamics. (Right) Fixed timestep of $1 e-3$ and three different meshes: mesh 1 corresponds to an initial mesh of meshsize of 1.25e-1, mesh 2 and mesh 3 are two successive uniform refinements of mesh 1 . Unlike the previous asymptotics, the effect of polynomial mismatch is reduced by decreasing the meshsize.

5.5. Sensitivity to Small Time-steps. We recall our earlier comment that (5.3) depends implicitly on the timestep $\delta t^{n+1}$. On the one hand, as $\delta t^{n+1} \downarrow 0,(5.3)$ approaches the explicit computation of curvature (5.8), which entails loss of accuracy as discussed previously (see for instance Figure 2.3). This is in conflict with the usual believe that small timesteps improve accuracy. On the other hand, since the position variation $\delta \mathbf{X}^{n+1}=\delta t^{n+1} \mathbf{V}^{n+1}$ satisfies

$$
\left\|\delta \mathbf{X}^{n+1}\right\|_{L^{\infty}\left(\Gamma^{n}\right)}=\delta t^{n+1}\left\|\mathbf{V}^{n+1}\right\|_{L^{\infty}\left(\Gamma^{n}\right)},
$$


requiring $\left|\delta \mathbf{X}^{n+1}\right|$ not to exceed locally $1 / 2$ of the meshsize (so as to avoid node crossing) imposes a smallness condition on the timesteps. This geometric restriction is different from the usual CFL condition, because it is unrelated to stability, but it has a similar effect. The resulting GC triple might not be sufficiently accurate.

The construction of schemes robust with respect to timestep size remains open.

\section{REFERENCES}

[1] E. Bänsch. Local mesh refinement in 2 and 3 dimensions. Impact Comput. Sci. Engrg., 3(3):181191, 1991.

[2] E. Bänsch. Finite element discretization of the Navier-Stokes equations with a free capillary surface. Numer. Math., 88(2):203-235, 2001.

[3] J. Barrett, H. Garcke, and R. Nürnberg. Parametric approximation of Willmore flow and related geometric evolution equations. SIAM J. Sci. Comput., 31(1):225-253, 2008.

[4] P. Binev, W. Dahmen, and R. DeVore. Adaptive finite element methods with convergence rates. Numer. Math., 97(2):219-268, 2004.

[5] A. Bonito, R. Nochetto, and M. Pauletti. Parametric FEM for geometric biomembranes. J. Comput. Phys., 229(9):3171-3188, 2010

[6] U. Clarenz, U. Diewald, G. Dziuk, M. Rumpf, and R. Rusu. A finite element method for surface restoration with smooth boundary conditions. Comput. Aided Geom. Design, 21(5):427445,2004

[7] K. Deckelnick, G. Dziuk, and C. M. Elliott. Computation of geometric partial differential equations and mean curvature flow. Acta Numer., 14:139-232, 2005.

[8] M. C. Delfour and J.-P. Zolésio. Shapes and geometries, volume 4 of Advances in Design and Control. Society for Industrial and Applied Mathematics (SIAM), Philadelphia, PA, 2001.

[9] A. Demlow. Higher-order finite element methods and pointwise error estimates for elliptic problems on surfaces. SIAM J. Numer. Anal., 47:805-827, 2009.

[10] G. Dziuk. Finite elements for the Beltrami operator on arbitrary surfaces. In Partial differential equations and calculus of variations, volume 1357 of Lecture Notes in Math., pages 142155. Springer, Berlin, 1988.

[11] G. Dziuk. An algorithm for evolutionary surfaces. Numer. Math., 58(6):603-611, 1991.

[12] G. Dziuk. Computational parametric willmore flow. Numer. Math., 111(1):55-80, 2008.

[13] D. Gilbarg and N. S. Trudinger. Elliptic partial differential equations of second order, volume 224 of Grundlehren der Mathematischen Wissenschaften [Fundamental Principles of Mathematical Sciences]. Springer-Verlag, Berlin, second edition, 1983.

[14] C. Heine. Isoparametric finite element approximations of curvature on hypersurfaces. Preprint from his webpage.

[15] D. Koster, O. Kriessl, and K. G. Siebert. Design of finite element tools for coupled surface and volume meshes. Numer. Math. Theor. Meth. Appl., 1(3):245-274, 2008).

[16] J. Maubach. Local bisection refinement for $n$-simplicial grids generated by reflection. SIAM J. Sci. Comput., 16(1):210-227, 1995.

[17] K. Mekchay, P. Morin, and R. H. Nochetto. AFEM for the Laplace-Beltrami operator on graphs: design and conditional contraction property. Math. Comp. (2010), to appear.

[18] S. Montiel and A. Ros. Curves and surfaces, volume 69 of Graduate Studies in Mathematics. American Mathematical Society, Providence, RI, 2005. Translated and updated from the 1998 Spanish edition by the authors.

[19] R. H. Nochetto, K. G. Siebert, and A. Veeser. Theory of adaptive finite element methods: an introduction. Multiscale, Nonlinear and Adaptive Approximation, pages 409-542, 2009.

[20] M. Pauletti. Parametric AFEM for Geometric Evolution Equations and Coupled Fluid-Membrane Interaction. $\mathrm{PhD}$ thesis, University of Maryland, 2008. http://www.lib.umd.edu/drum/handle/1903/8603.

[21] R. Rusu. An algorithm for the elastic flow of surfaces. Interfaces Free Bound., 7(3):229-239, 2005.

[22] J. Sokołowski and J.-P. Zolésio. Introduction to shape optimization, volume 16 of Springer Series in Computational Mathematics. Springer-Verlag, Berlin, 1992.

[23] M. Spivak. Calculus on manifolds. A modern approach to classical theorems of advanced calculus. W. A. Benjamin, Inc., New York-Amsterdam, 1965.

[24] R. Stevenson. The completion of locally refined simplicial partitions created by bisection. Math. Comp., 77(261):227-241 (electronic), 2008.

[25] J. Wloka. Partial differential equations. Cambridge University Press, Cambridge, 1987. 